Research Article

\title{
Determination of Modelling Error Statistics for Cold-Formed Steel Columns
}

\author{
R. P. Rokade $\mathbb{D}^{1}{ }^{1}$ K. Balaji Rao, ${ }^{2}$ and B. Palani ${ }^{3}$ \\ ${ }^{1}$ Tower Testing and Research Station, CSIR-Structural Engineering Research Centre, Chennai 600043, India \\ ${ }^{2}$ CSIR-Structural Engineering Research Centre, Chennai 600113, India \\ ${ }^{3}$ Department of Civil and Structural Engineering, Annamalai University, Annamalainagar, Chidambaram 608002, India
}

Correspondence should be addressed to R. P. Rokade; rokade@serc.res.in

Received 6 November 2019; Revised 3 January 2020; Accepted 6 January 2020; Published 28 February 2020

Academic Editor: Marco Corradi

Copyright ( 92020 R. P. Rokade et al. This is an open access article distributed under the Creative Commons Attribution License, which permits unrestricted use, distribution, and reproduction in any medium, provided the original work is properly cited.

In this article, an attempt has been made to estimate the Modelling Error (ME) associated with compression capacity models available in international standards for different failure modes of compression members fabricated from Cold-Formed Steel (CFS) lipped channel sections. For the first time, a database has been created using test results available in the literature for compression capacities of CFS lipped-channel sections. The database contains details of 273 numbers of compression member tests which have failed in different failure modes, namely, (i) flexural, torsional, flexural-torsional, local, and distortion buckling and (ii) failure by yielding. Only those sources, which report all the details, required to compute the capacities using different standards are included in the database. The results of experimental investigations carried out at CSIR-Structural Engineering Research Centre, Chennai, are also included in this test database. The international codes of practice used in calculation of compression capacities of the database columns considered in this paper are ASCE 10-15 (2015), AISI S100-16 (2016), AS/NZS 4600: 2018 (2018), and EN 19931-3:2006 (2006). The ASCE, AISI, AS/NZS, and EN design standards have different design guidelines with respect to the failure modes, e.g., ASCE 10-15 (2015) standard provides stringent criteria for maximum width to thickness ratio for stiffened and unstiffened elements. Hence, guidelines for the distortional buckling mode are not provided, whereas the AISI S100-16 (2016) and AS/NZS 4600: 2018 (2018) standards consider separate guidelines for distortional buckling mode and EN 1993-1-3:2006 (2006) standard considers combined local and distortional buckling mode. Further, the sample size for each design standard is varying depending on the design criteria and failure mode. Studies on statistical analysis of ME suggest that the compression capacity predicting models for flexural-torsional buckling mode are associated with large variation irrespective of the design standard. Similar observations are made for the flexural buckling model as per EN 1993-1-3:2006 (2018) standard and distortional buckling models as per AISI S100-16 (2016) and AS/NZS 4600: 2018 (2018) standards. The compression capacities for test database sections are evaluated by neglecting the partial safety factors available in design standards. The probabilistic analysis to determine statistical characteristics of compression capacity indicates the importance of consideration of $\mathrm{ME}$ as a random variable. Hence, the ME results will be useful in code calibration studies and may have potential reference to design practice.

\section{Introduction}

The exponential growth in all sectors, viz. industrial, housing, transportation, communication, services, has increased the demand of power supply by many folds. In order to cater to this increased demand, the transmission line towers have to support larger and heavier conductors in bundle configuration, Juette and Zaffanella [1]. This makes the tower configurations taller and wider, and hence, the tower system would become heavier. Conventionally, the transmission line towers are fabricated using Hot-Rolled Steel (HRS) angle sections. To reduce the tower weights, it is necessary either to consider eco-friendly alternate sustainable materials (viz., GFRP sections) or to produce lighter steel sections with higher strengths. Generally, CFS sections are used for achieving higher strengths for the structure and structural members without compromising on overall drift requirements. 
For CFS sections, the influence of cold work on mechanical properties of structural steel was investigated at Cornell University by Chajes et al. [2], Karren [3], Karren and Winter [4], and Winter and Uribe [5]. It was found that the changes in mechanical properties of CFS are due to cold stretching caused mainly by strain hardening and strain aging, occurring predominantly in the bent portions of the CFS sections. The effect of cold working increases the yield stress by a minimum of $15 \%$ [6].

Some of the advantages of CFS sections as against HRS sections are as follows:

(i) Cold rolling process produces any desired shape of cross section over longer lengths

(ii) High strength to weight ratio can be achieved in CFS sections

(iii) The connection methods that can be used for CFS sections are same as those for the HRS sections

(iv) Since CFS sections/members are lighter and stronger, they can be easily transported and erected

(v) Pregalvanised or precoated CFS sections have high resistance to corrosion

The abovementioned advantages of CFS members enable engineers to design cost effective transmission line towers. The CFS members can be fabricated to closely fit the design requirements. Recommendations for the design of plain and lipped angles, produced by cold-forming process for transmission towers, were given by Gaylord and Wilhoite [7]. These recommendations are included in ASCE 10-15 [8] standard. Some of the other international standards providing design guidelines for the use of CFS sections are AISI S100-16 [9], AS/ NZS 4600: 2018 [10], EN 1993-1-3:2006 [11], and IS 801 [12]. These codes recommend equations/models for prediction of compression and tension capacities of CFS members failing in different modes depending on geometrical, material, and boundary condition details. In this paper, the focus is on estimation of ME associated with equations specified in ASCE 10-15 [8], AISI S100-16 [9], AS/NZS 4600: 2018 [10], and EN 1993-1-3:2006 [11] standards for compression capacity estimation of CFS members. Some of the highlights of the international design standards considered are as follows. (i) The ASCE 10-15 [8] standard provides design guidelines for CFS sections for transmission line towers. In this standard, the CRC curve is used for the estimation of compression capacity without embedding any partial safety factor. (ii) The AISI S100-16 [9], AS/NZS 4600: 2018 [10], and EN 1993-1-3:2006 [11] standards are providing design guidelines for CFS section for general building structures.

The JCSS probabilistic model code [13] states that the models used in calculation of structural responses are usually not complete and exact, so the actual outcomes cannot be predicted without error. The variables used in model functions are associated with possible uncertainties which accounts for random effects that are neglected in the models, and uncertainties arise due to simplification in the mathematical relations.

As has been already pointed out, the focus of this paper is to establish the ME associated with the compression capacity formulae given in ASCE 10-15 [8], AISI S100-16 [9], AS/ NZS 4600: 2018 [10], and EN 1993-1-3:2006 [11] codes. In order to estimate the same, a database of experimental capacities of columns (pinned ended), made of CFS lipped channel sections failing in different modes, is created for the first time. This database includes experimental results for CFS lipped channel columns obtained from the literature along with the test results of three nominally similar columns tested at CSIR-SERC. A brief review of the literature on column tests, probabilistic analysis, and determination of reliability index for CFS lipped channel sections is presented here.

Number of researchers have conducted experiments on CFS lipped channel columns to study the influence of residual stress, cross-sectional dimensions, yield type (gradual or sharp) of stress strain curve, forming methods (press brake or cold rolling), local buckling, load application (concentric or eccentric), etc: Weng and Pekoz [14], Weng and Lin [15], Dat [16], Mulligan [17], Loughlan [18], Loughlan and Rhodes [19], Young and Rasmussen [20], Chilver [21], Pekoz [22], Zaras and Rhodes [23], Rokade et al. [24], Miller and Pekoz [25], Moldovan [26], Sivakumaran [27], Thomasson [28], Chou et al. [29], Pu et al. [30], Dundu [31]. The compression capacity results obtained from abovementioned experimental studies for CFS lipped channel sections are used in creation of database.

From the review of literature, it has been noted that recent research is directed towards experimental and analytical investigations on built-up CFS channel sections under axial compression. Ting et al. [32] have carried out numerical and experimental investigations to study the buckling behaviour of back to back built-up CFS channel sections covering stub columns to slender columns. The geometric imperfections were measured using equipment with LVDT $(0.01 \mathrm{~mm}$ accuracy) and considered in finite element modelling. It is observed that the FEA and test results were in good agreement and conservative with the calculated strengths as per AISI and AS/NZS standards for short, intermediate, and slender columns which failed through a combination of local and global buckling and/or global buckling. Whittle and Ramseyer [33] have conducted experimental compression tests on closedsection, built-up members formed of intermediately welded CFS channel sections, and the test capacities were compared to theoretical buckling capacities based on the AISI specifications modified slenderness ratio. Use of the modified slenderness ratio was exceedingly conservative. Capacities based on the unmodified slenderness ratio provisions were less conservative. Ye et al. [34, 35] presented the results of experimental and numerical investigations carried out on CFS plain and lipped channels under axial compression to study the interaction of local and overall flexural buckling. The measured initial geometric imperfections and material properties are used in development of finite element models. The finite element and experimental results were compared to the compression capacity calculated using Eurocode and direct strength method. It was observed that the Eurocode provides conservative predictions for the compressive capacity of plain and lipped channel sections, while the direct strength method predictions are more accurate for lipped 
channels. It has been stated that these studies represent the state of the art.

Balaji Rao and Appa Rao [36] carried out studies on probabilistic analysis of strength of steel imperfect columns. Based on these studies, a characteristic strength equation is proposed which will be useful in rational design of imperfect columns. The researchers have estimated ratios of load carrying capacity to the yield load of column (ME) using CRC, AISC-ASD, AISC-PD, AISC-LRFD, SSRC Curve 2, IS 800: 1984, Lui and Chen [37], and Rondal and Maquio [38] formulae/model functions.

The determination of reliability index for CFS elements or members is presented in several research reports of the University of Missouri-Rolla [39-43] and Supomsilaphachai et al. [44], where both the basic research data as well as the reliability index inherent in the AISI Specification are presented in great detail. The entire set of data for HR steel and CFS, was reanalysed by Ellingwood et al. [45]; Galambos et al. [46]; and Ellingwood et al. [47] using (a) updated load statistics and (b) a more advanced level of probability analysis which was able to incorporate probability distributions and to describe the true distributions more realistically.

From the review of the literature, presented above, it is found that studies dealing with ME estimation for CFS compression members are scanty. However, while estimating the ME care is taken to neglect the use of any partial factors embedded in the codal equation. The main objectives of this paper are (i) creation of database for compression capacities of CFS lipped-channel sections from the test results available in literature, (ii) estimation of ME for database results for the various failure modes as per ASCE 10-15 [8], AISI S100-16 [9], AS/NZS 4600:2018 [10], and EN 1993-1-3: 2006 [11] standards, (iii) studies on statistical characteristics of ME estimated at step (ii), and (iv) fitting a statistical distribution for the ME.

The details of experimental investigations carried out at CSIR-Structural Engineering Research Centre, Chennai, which included in test database are provided in next section.

\section{Compression Tests on CFS Lipped Channels Carried Out at CSIR-SERC}

At CSIR-SERC, Chennai, experimental investigations were carried out to study the buckling behaviour and to evaluate the compression capacity of axially loaded CFS lipped channel compression members (leg member of a X-braced tower panel). The scope of these experimental investigations consist of measurement of sectional dimensions for the procured lipped channels to study the imperfection associated with the CFS sections, coupon tests for determining the mechanical properties of CFS, and element level studies on axially loaded compression members to observe the buckling failure mode and to evaluate the capacity. The details for abovementioned experimental investigations are summarised as below:

2.1. Sectional Dimensions. The sectional dimensions are verified for each specimen of $3.0 \mathrm{~m}$ length with the help of
Vernier calliper (least count $0.01 \mathrm{~mm}$ ) at seven locations (starting from one end at $0.0 \mathrm{~m}$ to every $0.5 \mathrm{~m}$ ) along the length, and thicknesses are verified using micrometer screw gauge (least count $0.001 \mathrm{~mm}$ ) at both the ends. The details of dimension measurements for two number of sample specimens are given in Table 1. The tolerance limit for profile dimensions as per IS: $811-1987$ [48] is $\pm 0.5 \mathrm{~mm}$ and for the thickness of strip used in making of cold formed section is $\pm 0.5 \mathrm{~mm}$ as per IS: $1852-1985$ [49]. It is observed that the sectional dimensions and member thicknesses are within the tolerance limits. To measure the straightness, the specimens were placed horizontally on a plane surface and a long precision ruler was used as a reference plane.

2.2. Coupon Tests. The material properties were determined by tensile coupon tests. The coupons were cut from the centre of web of CFS lipped channel sections of the same batch as per ASTM Standard E8/E8M-15a [50] and tested as per ASTM Standard E6-15 [51] in a 100 Ton Universal Testing Machine (UTM). An extensometer of $50 \mathrm{~mm}$ gauge length was used to measure the longitudinal strain. The displacement control for load application is kept at $0.5 \mathrm{~mm} /$ min, and the inbuilt data acquisition system of UTM with $1 \mathrm{~Hz}$ sampling frequency was used to record the load and strain values during the test. The stress-strain curve obtained along with the coupon specimen details are presented in Figure 1. As the material is gradually yielding material, the yield stress was determined by the $0.2 \%$ offset method [52]. The tension coupon test set up for CFS and the coupon test specimen after failure is shown in Figure 2.

2.3. Element-Level Tests. The element-level compression tests were carried out on lipped channel sections LC $90 \times 50 \times 20 \times 3.15 \mathrm{~mm}$ (Lipped Channel - Web depth $\times$ Flange width $\times$ Lip depth $\times$ Thickness). The tests were categorized as concentrically loaded members corresponding to leg members in a latticed tower panel. The tests were conducted using 250 Ton capacity displacement control UTM with spherical joints and ball bearing head at the top end and fixed base at the bottom. The CFS lipped channel specimens fabricated were of $2.225 \mathrm{~m}$ in length and connected with specially made fixtures at ends using 3 numbers of $16 \mathrm{~mm}$ diameter bolts as shown in Figure 3. This is in order to simulate the exact member end condition for leg member of a X-braced panel. During the tests, the lateral displacements of flanges and web were measured using displacement dial gauges $(0.01 \mathrm{~mm}$ least count). The linear foil strain gauges were instrumented across the cross section of specimens to measure the longitudinal strain variations throughout the load application. The test responses for dial and strain gauges were acquired using HBM data logger. In order to measure the strains and displacement responses at critical location, specimens were instrumented at maximum lateral displacement sections, i.e., mid length of the specimens. The test set-up and the three tested specimen's photos are shown in Figure 4. As the end fixtures are made of $16 \mathrm{~mm}$ thick HRS plates and the specimen thickness is $3.15 \mathrm{~mm}$, the stiffness of end fixtures is higher compared to the specimen. Hence, it is 
TABLE 1: Dimension measurement details of two number of CFS lipped channel specimens.

\begin{tabular}{|c|c|c|c|c|c|c|c|c|c|}
\hline Specimen & Section No & Distance & Web $(\mathrm{mm})$ & Flange1 $(\mathrm{mm})$ & Flange2 $(\mathrm{mm})$ & Lip1 (mm) & Lip2 (mm) & Thk1 (mm) & Thk2 (mm) \\
\hline \multirow{8}{*}{1} & 1 & 0.00 & 90.42 & 50.00 & 49.79 & 19.90 & 20.50 & 3.25 & 3.25 \\
\hline & 2 & 0.50 & 89.77 & 50.03 & 50.03 & 20.24 & 20.45 & - & - \\
\hline & 3 & 1.00 & 90.26 & 50.36 & 50.29 & 19.68 & 20.43 & - & - \\
\hline & 4 & 1.50 & 89.75 & 50.44 & 50.47 & 19.58 & 19.55 & - & - \\
\hline & 5 & 2.00 & 89.91 & 49.96 & 50.40 & 19.60 & 19.86 & - & - \\
\hline & 6 & 2.50 & 89.94 & 50.29 & 50.34 & 19.52 & 19.55 & - & - \\
\hline & 7 & 3.00 & 90.47 & 49.65 & 50.50 & 20.09 & 20.24 & 3.25 & 3.40 \\
\hline & \multicolumn{2}{|c|}{ Average } & 90.07 & 50.10 & 50.26 & 19.80 & 20.08 & 3.25 & 3.33 \\
\hline \multirow{8}{*}{2} & 1 & 0.00 & 89.52 & 50.44 & 49.70 & 20.05 & 19.54 & 3.22 & 3.20 \\
\hline & 2 & 0.50 & 90.46 & 50.46 & 49.80 & 20.05 & 19.60 & - & - \\
\hline & 3 & 1.00 & 90.26 & 50.30 & 50.44 & 20.25 & 19.80 & - & - \\
\hline & 4 & 1.50 & 90.39 & 50.18 & 49.93 & 20.22 & 19.94 & - & - \\
\hline & 5 & 2.00 & 89.52 & 49.81 & 50.03 & 19.77 & 19.81 & - & - \\
\hline & 6 & 2.50 & 89.64 & 49.89 & 49.58 & 19.70 & 20.44 & - & - \\
\hline & 7 & 3.00 & 90.04 & 49.55 & 49.93 & 19.86 & 19.56 & 3.17 & 3.60 \\
\hline & \multicolumn{2}{|c|}{ Average } & 89.98 & 50.09 & 49.92 & 19.98 & 19.81 & 3.20 & 3.40 \\
\hline \multicolumn{3}{|c|}{ Minimum dimension required } & 90.00 & 50.00 & 50.00 & 20.00 & 20.00 & 3.15 & 3.15 \\
\hline
\end{tabular}

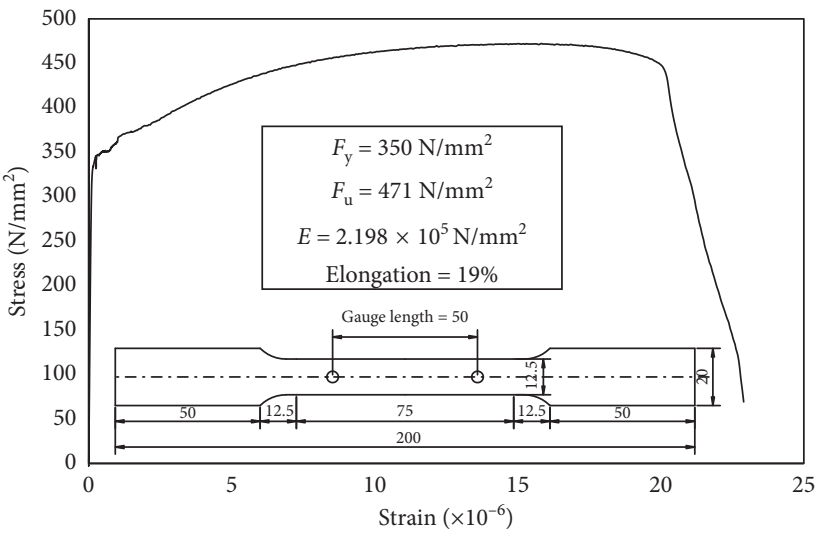

Figure 1: Stress-Strain plot and tension coupon details.

assumed that the end condition of test specimen will act as pin ends. Accordingly, the effective length for analysis is considered between centre to centre of the end connection. The test specimens have failed in flexural-torsional buckling mode and the observed test capacities are 119, 118, and $113 \mathrm{kN}$ with an average compression capacity of $116.67 \mathrm{kN}$. The capacities calculated using the assumed effective length and as per ASCE 10-15 [8] and AISI S100-16 [9], AS/NZS 4600:2018 [10] are in good agreement with the test capacity. This observation shows that the effective length determined assuming pinned end conditions is satisfactory. However, it is to be noted that exact determination of effective length is difficult. The results of test capacities and codal predictions for the test specimens are presented in Table 2 . The capacity predictions are based on the design standards under consideration and calculated for effective length equal to the centre to centre of bolted connection $(1915 \mathrm{~mm})$ and without considering the safety or partial safety factors present in model equations.

The specimen details and results of above compression tests along with results of compression tests available in the literature for CFS lipped channel pinned columns considered in creation of database is presented in the next section.

\section{Database of Compression Capacity for CFS Lipped Channel Members}

In this study, for the first time, a test database is created from the experimental details available in the literature for the CFS lipped channel compression members. Table 3 provides the details of database created. Further, it gives information on the test data exclusion criteria used in filtering the test data of each study considered. It also indicates the reference data number for particular referenced compression members from the detailed test database presented in Table 4 and Figure 5.

The selection of test compression members, obtained from the literature, for the ME estimation was based on the following criteria:

(1) The member is a CFS lipped channel section

(2) The test results are close to the calculated capacities ( $\pm \%$ variations used)

(3) The member is concentrically loaded during the test

(4) The member tested with pinned end condition on both ends

(5) The member is not having any perforations along the length

(6) The member is not having any intermediate stiffeners in web

A total number of 577 compression members' test details available in the literature are used in the creation of test database. Out of this 273 compression members (120 columns and 153 stub columns) are filtered using abovementioned criteria and used in test database. The detailed test database (refer Table 4 and Figure 5) includes test compression member details like out-to-out cross-section dimensions (lipped channel section web depth, flange width, and lip depth), thickness of section, corner radius (inner), length of the member, material properties (Young's modulus, yield, and ultimate strength), test load, type of compression member (column or stub column), torsion 


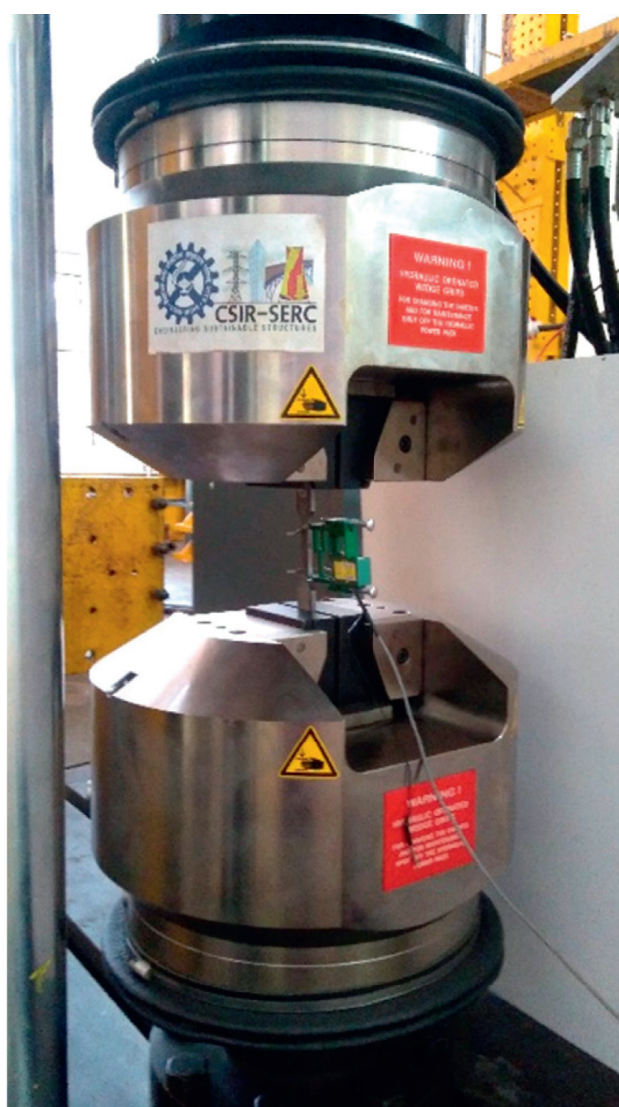

(a)

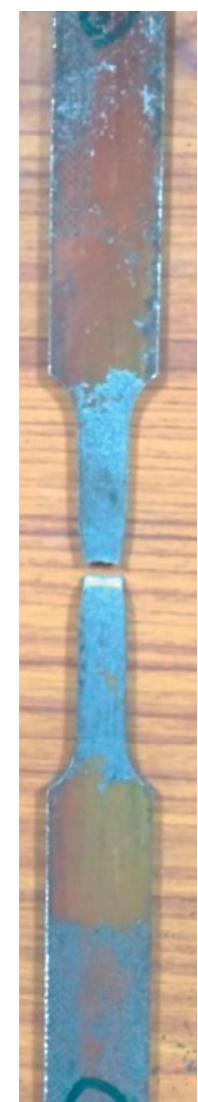

(b)

Figure 2: Test details of coupon tests carried out in this investigation. (a) Test set-up (b) Tested specimens.

indicator, and forming method (press brake or roll forming). While creating the test database, it is noticed that not all the required details were available and were assumed appropriately as follows:

(a) In some of the references, the material properties like Young's modulus and/or ultimate strength details were not available which were taken from the standards based on the value of yield strength and country/region in which the tests were conducted.

(b) The pinned end condition was assumed at both ends for some of the compression members for which the details were not available.

(c) For the compression members where it was not clearly mentioned that the torsional buckling is allowed, it is assumed to be allowed. This assumption provides calculated results in a more realistic behaviour.

(d) In one of the references, the member length was not indicated for the stub columns. Though it is not required for calculating the section capacity, the same was assumed as three times the web depth. This is just to indicate in the test database.
Further, to estimate ME, initially the test database for CFS lipped channel compression members has been filtered through the geometric proportion criteria stipulated for each code separately which is explained in next section. Then the database compression members are further filtered for the limiting maximum slenderness ratio. The limiting maximum slenderness ratio for tower leg members (axially loaded compression members in transmission tower) is given as 120 in ASCE standard. Though this limitation is not present in AISI, AS/NZS, and EN standards, the same is applied in this study as ASCE standard provides design guidelines for "Design of Latticed Steel Transmission Structures," whereas the other standards are applicable for general steel structures. This philosophy of limiting slenderness ratio to the axially loaded compression members is based on the fact that the overall deformation of transmission line towers is governed by the stiffness of leg members. Large deformations resulting with higher slenderness ratios of lower stiffness members may cause disturbance to the safe clearance limits of electrical conductors with the tower profile.

Before estimating the ME associated with different codal equations, a brief overview of codal clauses that would help in discussing the results of statistical analysis are presented in the next section. 

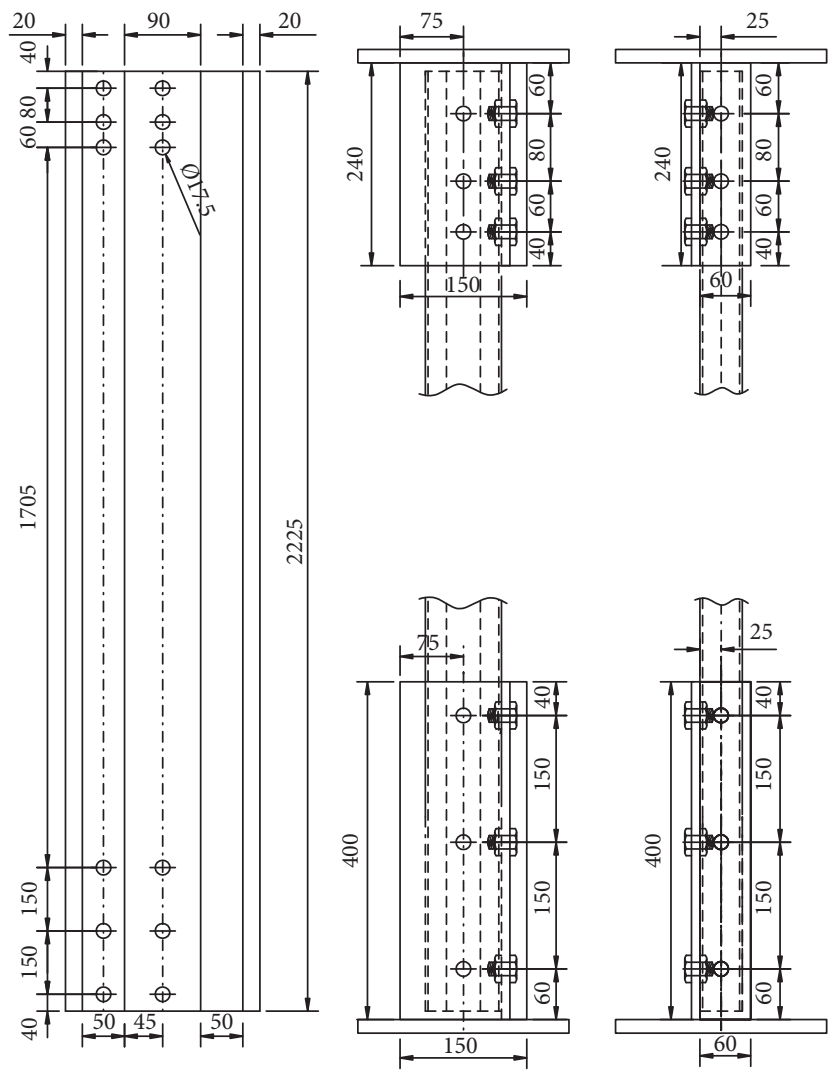

Note - All dimension are in $\mathrm{mm}$

Figure 3: Fabrication details of the compression element and details of the end fixtures.

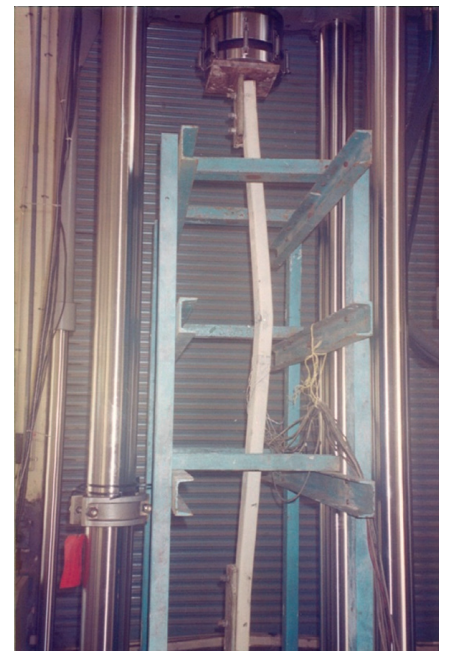

(a)

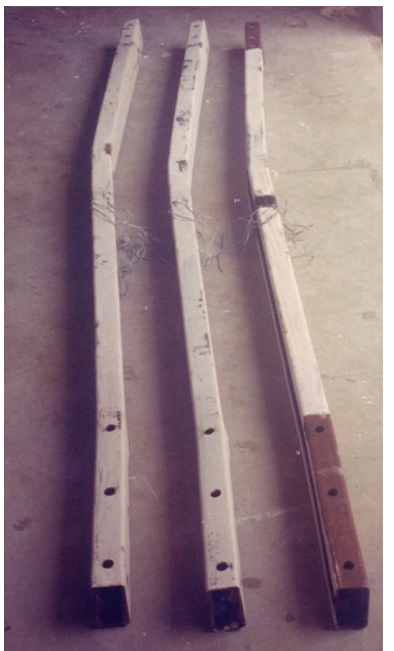

(b)

FIgURE 4: Test details of compression tests carried out in this investigation. (a) Test set-up (b) Tested specimens.

\section{Codal Provisions for Estimation of Compression Capacity of CFS Columns}

In this section, a brief review of design guidelines for CFS sections available in International design standards, i.e.,
ASCE 10-15 [8], AISI S100-16 [9], AS/NZS 4600: 2018 [10], and EN 1993-1-3:2006 [11] are presented (refer Table 5). The design guidelines of AISI and AS/NZS standards are similar and hence presented in a single column (refer Table 5). The design guidelines covered here focus on increase in strength 
TABLE 2: Results of experimental investigation, column capacity in torsional-flexural buckling mode.

\begin{tabular}{|c|c|c|c|c|}
\hline Design standard & $\begin{array}{c}\text { Calculated compression } \\
\text { capacity }(\mathrm{kN})\end{array}$ & $\begin{array}{l}\text { Average test compression } \\
\text { capacity }(\mathrm{kN})\end{array}$ & $\begin{array}{c}\text { Difference in compression } \\
\text { capacity }(\mathrm{kN})\end{array}$ & $\begin{array}{c}\% \\
\text { Difference }\end{array}$ \\
\hline ASCE 10-15 (2015) & 120.14 & & 3.47 & 2.97 \\
\hline $\begin{array}{l}\text { AISI S100-16 (2016) \& AS/NZS } \\
\text { 4600: } 2018 \text { (2018) }\end{array}$ & 112.84 & 116.67 & -3.83 & -3.28 \\
\hline EN 1993-1-3:2006 (2006) & 98.34 & & -18.33 & -15.71 \\
\hline
\end{tabular}

TABLE 3: Test database for cold-formed lipped channel compression members.

\begin{tabular}{|c|c|c|c|c|c|c|c|c|}
\hline \multirow{3}{*}{ Sr. no. } & \multirow{3}{*}{ Author(s) } & \multicolumn{5}{|c|}{ No. of compression members } & \multirow{3}{*}{ Criterion $^{\#}$} & \multirow{3}{*}{ Ref. data no. in database } \\
\hline & & \multirow[t]{2}{*}{ Year } & \multicolumn{2}{|c|}{ Tested } & \multicolumn{2}{|c|}{ Data used in this study } & & \\
\hline & & & Column & Stub column & Column & Stub column & & \\
\hline 1 & Weng and Pekoz & 1990 & 68 & 25 & 30 & 13 & 1 & 1 to 43 \\
\hline 2 & Dat & 1980 & 60 & 20 & 26 & 11 & 1,2 & 44 to 69 and 263 to 273 \\
\hline 3 & Mulligan & 1983 & 21 & 36 & 13 & 25 & 1,3 & 70 to 81,196 and 171 to 195 \\
\hline 4 & Loughlan and Rhodes & 1979,1980 & 33 & - & 7 & - & 3 & 82 to 88 \\
\hline 5 & Young@ & 1998 & 15 & 6 & 10 & 2 & $2,3,4$ & 89 to 100 \\
\hline 6 & Rokade et al. & 2010 & 3 & - & 3 & - & - & 101 to 103 \\
\hline 7 & Miller and Pekoz & 1994 & - & 31 & - & 18 & 5 & 104 to 121 \\
\hline 8 & Moldavan & 1994 & 37 & 27 & 18 & 11 & 1 & 121 to 139 and 140 to 150 \\
\hline 9 & Sivakumaran & 1987 & - & 48 & - & 6 & 5 & 151 to 156 \\
\hline 10 & Thomasson & 1978 & 46 & - & 14 & - & 6 & 157 to 170 \\
\hline 11 & Chilvers $\$$ & 1954 & - & 28 & - & 16 & 1 & 197 to 212 \\
\hline 12 & Pekoz $^{\$}$ & 1977 & - & 19 & - & 12 & 1 & 213 to 224 \\
\hline 13 & Zaras and Rhodes* & 1987 & - & 6 & - & 2 & 1 & 225 to 226 \\
\hline 14 & $\mathrm{Pu}$ & 1998 & - & 36 & - & 36 & - & 227 to 262 \\
\hline 15 & Dundu & 2014 & 9 & 3 & 9 & 3 & - & 273 to 285 \\
\hline
\end{tabular}

${ }^{\#}$ Criterion used to exclude some of the test compression members given in reference. ${ }^{\circledR}$-Some of the test compression members of this reference are considered in test database, finally they were excluded based on very high ME or no match in calculated and experimental results. ${ }^{\$}-$ The data are taken from Mulligan [17] as the original reference is not available. ${ }^{*}$ The data are taken from Chou et al. [29] as the original reference is not available.

due to cold working, limiting geometrical proportions, and column capacity prediction models for different failure modes.

Considering the above codal provisions, the database CFS lipped channel sections are filtered for limiting criteria, and then the compression capacities are calculated for the estimation of ME. The ME estimation procedure and statistical properties of ME for database CFS lipped channel sections is presented in the next section.

\section{Modelling Error Estimation Methodology}

The mathematical relations or model functions used to predict the capacity of the compression member are based on the simplifying assumptions and/or neglected random effects for some of the parameters involved in the prediction equation [13]. Hence, the model function may not completely describe the physical phenomenon under consideration. This in combination with lack of complete knowledge of the modeller causes the difference in the actual and predicted compression member capacities. The difference in capacity can be quantified using ME analysis. Accordingly, the ME should be defined as the ratio of actual capacity to calculated capacity. It may be possible that the test capacity may not be the true capacity as there may be some unavoidable errors involve in the test and measurement procedure, but even then, it provides close estimate to the true capacity. In this study, the ME is taken as the ratio of test to calculated capacity of compression member. Further, in ME analysis, the model functions are considered for various modes of failure viz. flexure, flexure-torsion, local and distortion buckling, and yielding of section based on ASCE 10-15 [8], AISI S100-16 [9], AS/NZS 4600: 2018 [10], and EN 1993-1-3:2006 [11] standards as described in above section.

Accordingly, after passing through the filtering criteria as discussed in "Database of Compression Capacity for CFS Lipped Channel Members", the member compression capacity has been calculated for the balance test database members for governing failure modes given in each design standard after removing the safety or partial safety factors present in the model function. Then the ME estimated taking the ratio of test capacity as per database with the calculated capacity. The results of calculated ME and its statistical properties for the test database compression members in various failure modes as per abovementioned design standards are presented in Table 6. The histograms of ME for various failure modes with respect to design standards are shown in Figures 6-8 . Further, Chi-square goodness-of-fit test has been performed on ME for selection of statistical distributions, and the results of this test are provided in Table 7. The failure modes considered for column members are flexure, flexure-torsion, and local and distortion buckling and for stub columns failure by yielding and local and distortion buckling. 
TABLE 4: Test database of axially loaded cold-formed steel lipped channel section compression members.

\begin{tabular}{|c|c|c|c|c|c|c|c|c|c|c|c|c|c|}
\hline $\begin{array}{l}\text { S. } \\
\text { No. }\end{array}$ & $\begin{array}{c}W \\
(\mathrm{~mm})\end{array}$ & $\begin{array}{c}F \\
(\mathrm{~mm})\end{array}$ & $\begin{array}{c}L \\
(\mathrm{~mm})\end{array}$ & $\begin{array}{c}T \\
(\mathrm{~mm})\end{array}$ & $\begin{array}{c}R \\
(\mathrm{~mm})\end{array}$ & $\begin{array}{l}\text { Length } \\
(\mathrm{mm})\end{array}$ & $\begin{array}{c}F_{\mathrm{y}}(\mathrm{N} / \\
\left.\mathrm{mm}^{2}\right)\end{array}$ & $\begin{array}{c}F_{\mathrm{u}}(\mathrm{N} / \\
\left.\mathrm{mm}^{2}\right)\end{array}$ & $\begin{array}{l}P_{\text {Test }} \\
(\mathrm{kN})\end{array}$ & $\begin{array}{l}E(\mathrm{~N} / \\
\left.\mathrm{mm}^{2}\right)\end{array}$ & $\begin{array}{l}\text { Type of } \\
\text { comp. } \\
\text { member }\end{array}$ & $\begin{array}{l}\text { Torsion } \\
\text { allowed }\end{array}$ & $\begin{array}{l}\text { Forming } \\
\text { method }\end{array}$ \\
\hline 1 & 80.06 & 41.86 & 17.93 & 3.02 & 3.96 & 254.00 & 278.22 & 366.00 & 166.43 & 203.255 & SC & $\mathrm{Y}$ & $\mathrm{R}$ \\
\hline 2 & 80.06 & 41.86 & 17.93 & 3.02 & 3.96 & 685.80 & 278.22 & 366.00 & 143.74 & 203.255 & $\mathrm{C}$ & $\mathrm{Y}$ & $\mathrm{R}$ \\
\hline 3 & 80.06 & 41.86 & 17.93 & 3.02 & 3.96 & 990.60 & 278.22 & 366.00 & 134.84 & 203.255 & $\mathrm{C}$ & $\mathrm{Y}$ & $\mathrm{R}$ \\
\hline 4 & 80.06 & 41.86 & 17.93 & 3.02 & 3.96 & 1295.40 & 278.22 & 366.00 & 126.83 & 203.255 & $\mathrm{C}$ & $\mathrm{Y}$ & $\mathrm{R}$ \\
\hline 5 & 80.06 & 41.86 & 17.93 & 3.02 & 3.96 & 1600.20 & 278.22 & 366.00 & 87.67 & 203.255 & $\mathrm{C}$ & $\mathrm{Y}$ & $\mathrm{R}$ \\
\hline 6 & 77.90 & 41.38 & 18.16 & 2.44 & 3.96 & 254.00 & 357.25 & 428.63 & 155.75 & 203.255 & SC & $\mathrm{Y}$ & $\mathrm{R}$ \\
\hline 7 & 77.90 & 41.38 & 18.16 & 2.44 & 3.96 & 254.00 & 357.25 & 428.63 & 161.98 & 203.255 & SC & $\mathrm{Y}$ & $\mathrm{R}$ \\
\hline 8 & 77.90 & 41.38 & 18.16 & 2.44 & 3.96 & 685.80 & 357.25 & 428.63 & 134.39 & 203.255 & $\mathrm{C}$ & $\mathrm{Y}$ & $\mathrm{R}$ \\
\hline 9 & 77.90 & 41.38 & 18.16 & 2.44 & 3.96 & 990.60 & 357.25 & 428.63 & 129.94 & 203.255 & $\mathrm{C}$ & $\mathrm{Y}$ & $\mathrm{R}$ \\
\hline 10 & 77.90 & 41.38 & 18.16 & 2.44 & 3.96 & 1295.40 & 357.25 & 428.63 & 105.91 & 203.255 & $\mathrm{C}$ & $\mathrm{Y}$ & $\mathrm{R}$ \\
\hline 11 & 77.90 & 41.38 & 18.16 & 2.44 & 3.96 & 1600.20 & 357.25 & 428.63 & 75.65 & 203.255 & $\mathrm{C}$ & $\mathrm{Y}$ & $\mathrm{R}$ \\
\hline 12 & 76.15 & 44.60 & 17.63 & 1.91 & 5.56 & 254.00 & 379.57 & 527.43 & 130.83 & 203.255 & SC & $\mathrm{Y}$ & $\mathrm{R}$ \\
\hline 13 & 76.15 & 44.60 & 17.63 & 1.91 & 5.56 & 254.00 & 379.57 & 527.43 & 133.06 & 203.255 & SC & $\mathrm{Y}$ & $\mathrm{R}$ \\
\hline 14 & 76.15 & 44.60 & 17.63 & 1.91 & 5.56 & 254.00 & 379.57 & 527.43 & 131.72 & 203.255 & SC & $\mathrm{Y}$ & $\mathrm{R}$ \\
\hline 15 & 76.15 & 44.60 & 17.63 & 1.91 & 5.56 & 685.80 & 379.57 & 527.43 & 112.59 & 203.255 & $\mathrm{C}$ & $\mathrm{Y}$ & $\mathrm{R}$ \\
\hline 16 & 76.15 & 44.60 & 17.63 & 1.91 & 5.56 & 982.98 & 379.57 & 527.43 & 99.24 & 203.255 & $\mathrm{C}$ & $\mathrm{Y}$ & $\mathrm{R}$ \\
\hline 17 & 76.15 & 44.60 & 17.63 & 1.91 & 5.56 & 1295.40 & 379.57 & 527.43 & 72.98 & 203.255 & $\mathrm{C}$ & $\mathrm{Y}$ & $\mathrm{R}$ \\
\hline 18 & 76.15 & 44.60 & 17.63 & 1.91 & 5.56 & 1600.20 & 379.57 & 527.43 & 56.52 & 203.255 & $\mathrm{C}$ & $\mathrm{Y}$ & $\mathrm{R}$ \\
\hline 19 & 76.15 & 44.60 & 17.63 & 1.91 & 5.56 & 1917.70 & 379.57 & 527.43 & 43.17 & 203.255 & $\mathrm{C}$ & $\mathrm{Y}$ & $\mathrm{R}$ \\
\hline 20 & 76.84 & 41.15 & 15.49 & 2.21 & 3.96 & 254.00 & 264.58 & 348.36 & 104.58 & 203.255 & SC & $\mathrm{Y}$ & $\mathrm{P}$ \\
\hline 21 & 76.84 & 41.15 & 15.49 & 2.21 & 3.96 & 254.00 & 264.58 & 348.36 & 104.58 & 203.255 & SC & $\mathrm{Y}$ & $\mathrm{P}$ \\
\hline 22 & 76.84 & 41.15 & 15.49 & 2.21 & 3.96 & 680.72 & 264.58 & 348.36 & 80.10 & 203.255 & $\mathrm{C}$ & $\mathrm{Y}$ & $\mathrm{P}$ \\
\hline 23 & 76.84 & 41.15 & 15.49 & 2.21 & 3.96 & 990.60 & 264.58 & 348.36 & 77.88 & 203.255 & $\mathrm{C}$ & $\mathrm{Y}$ & $\mathrm{P}$ \\
\hline 24 & 76.84 & 41.15 & 15.49 & 2.21 & 3.96 & 1295.40 & 264.58 & 348.36 & 71.20 & 203.255 & $\mathrm{C}$ & $\mathrm{Y}$ & $\mathrm{P}$ \\
\hline 25 & 76.23 & 41.45 & 15.37 & 1.80 & 3.96 & 254.00 & 250.11 & 344.98 & 82.33 & 203.255 & SC & $\mathrm{Y}$ & $\mathrm{P}$ \\
\hline 26 & 76.23 & 41.45 & 15.37 & 1.80 & 3.96 & 254.00 & 250.11 & 344.98 & 78.77 & 203.255 & SC & $\mathrm{Y}$ & $\mathrm{P}$ \\
\hline 27 & 76.23 & 41.45 & 15.37 & 1.80 & 3.96 & 254.00 & 250.11 & 344.98 & 79.21 & 203.255 & SC & $\mathrm{Y}$ & $\mathrm{P}$ \\
\hline 28 & 76.23 & 41.45 & 15.37 & 1.80 & 3.96 & 685.80 & 250.11 & 344.98 & 71.65 & 203.255 & $\mathrm{C}$ & $\mathrm{Y}$ & $\mathrm{P}$ \\
\hline 29 & 76.23 & 41.45 & 15.37 & 1.80 & 3.96 & 990.60 & 250.11 & 344.98 & 69.42 & 203.255 & $\mathrm{C}$ & $\mathrm{Y}$ & $\mathrm{P}$ \\
\hline 30 & 76.23 & 41.45 & 15.37 & 1.80 & 3.96 & 1295.40 & 250.11 & 344.98 & 57.85 & 203.255 & $\mathrm{C}$ & $\mathrm{Y}$ & $\mathrm{P}$ \\
\hline 31 & 76.23 & 41.45 & 15.37 & 1.80 & 3.96 & 1600.20 & 250.11 & 344.98 & 49.84 & 203.255 & $\mathrm{C}$ & $\mathrm{Y}$ & $\mathrm{P}$ \\
\hline 32 & 76.23 & 41.45 & 15.37 & 1.80 & 3.96 & 1905.00 & 250.11 & 344.98 & 43.17 & 203.255 & $\mathrm{C}$ & $\mathrm{Y}$ & $\mathrm{P}$ \\
\hline 33 & 127.94 & 63.27 & 22.30 & 3.00 & 3.18 & 431.80 & 210.77 & 351.05 & 201.59 & 203.255 & SC & $\mathrm{Y}$ & $\mathrm{P}$ \\
\hline 34 & 127.94 & 63.27 & 22.30 & 3.00 & 3.18 & 1397.00 & 210.77 & 351.05 & 152.19 & 203.255 & $\mathrm{C}$ & $\mathrm{Y}$ & $\mathrm{P}$ \\
\hline 35 & 127.94 & 63.27 & 22.30 & 3.00 & 3.18 & 1905.00 & 210.77 & 351.05 & 135.28 & 203.255 & $\mathrm{C}$ & $\mathrm{Y}$ & $\mathrm{P}$ \\
\hline 36 & 127.94 & 63.27 & 22.30 & 3.00 & 3.18 & 2286.00 & 231.50 & 357.66 & 123.71 & 203.255 & $\mathrm{C}$ & $\mathrm{Y}$ & $\mathrm{P}$ \\
\hline 37 & 127.94 & 63.27 & 22.30 & 3.00 & 3.18 & 2794.00 & 231.50 & 357.66 & 99.24 & 203.255 & $\mathrm{C}$ & $\mathrm{Y}$ & $\mathrm{P}$ \\
\hline 38 & 67.18 & 34.98 & 15.82 & 1.63 & 2.39 & 254.00 & 230.47 & 276.50 & 58.74 & 203.255 & SC & $\mathrm{Y}$ & $\mathrm{P}$ \\
\hline 39 & 67.18 & 34.98 & 15.82 & 1.63 & 2.39 & 787.40 & 230.47 & 276.50 & 49.84 & 203.255 & $\mathrm{C}$ & $\mathrm{Y}$ & $\mathrm{P}$ \\
\hline 40 & 67.18 & 34.98 & 15.82 & 1.63 & 2.39 & 1041.40 & 230.47 & 276.50 & 46.28 & 203.255 & $\mathrm{C}$ & $\mathrm{Y}$ & $\mathrm{P}$ \\
\hline 41 & 67.18 & 34.98 & 15.82 & 1.63 & 2.39 & 1320.80 & 220.89 & 310.67 & 35.60 & 203.255 & $\mathrm{C}$ & $\mathrm{Y}$ & $\mathrm{P}$ \\
\hline 42 & 67.18 & 34.98 & 15.82 & 1.63 & 2.39 & 1574.80 & 220.89 & 310.67 & 30.71 & 203.255 & $\mathrm{C}$ & $\mathrm{Y}$ & $\mathrm{P}$ \\
\hline 43 & 67.18 & 34.98 & 15.82 & 1.63 & 2.39 & 1752.60 & 220.89 & 310.67 & 27.59 & 203.255 & $\mathrm{C}$ & $\mathrm{Y}$ & $\mathrm{P}$ \\
\hline 44 & 44.20 & 42.93 & 18.92 & 2.29 & 3.94 & 2209.80 & 305.02 & 434.37 & 40.18 & 203.255 & $\mathrm{C}$ & $\mathrm{Y}$ & $\mathrm{R}$ \\
\hline 45 & 44.20 & 42.93 & 18.92 & 2.29 & 3.94 & 1905.00 & 305.02 & 434.37 & 54.29 & 203.255 & $\mathrm{C}$ & $\mathrm{Y}$ & $\mathrm{R}$ \\
\hline 46 & 44.20 & 42.93 & 18.92 & 2.29 & 3.94 & 1752.60 & 305.02 & 434.37 & 59.41 & 203.255 & $\mathrm{C}$ & $\mathrm{Y}$ & $\mathrm{R}$ \\
\hline 47 & 44.20 & 42.93 & 18.92 & 2.29 & 3.94 & 1600.20 & 305.02 & 434.37 & 71.20 & 203.255 & $\mathrm{C}$ & $\mathrm{Y}$ & $\mathrm{R}$ \\
\hline 48 & 44.20 & 42.93 & 18.92 & 2.29 & 3.94 & 1447.80 & 305.02 & 434.37 & 89.00 & 203.255 & $\mathrm{C}$ & $\mathrm{Y}$ & $\mathrm{R}$ \\
\hline 49 & 44.20 & 42.93 & 18.92 & 2.29 & 3.94 & 1295.40 & 305.02 & 434.37 & 102.35 & 203.255 & $\mathrm{C}$ & $\mathrm{Y}$ & $\mathrm{R}$ \\
\hline 50 & 44.20 & 42.93 & 18.92 & 2.29 & 3.94 & 1143.00 & 305.02 & 434.37 & 109.03 & 203.255 & $\mathrm{C}$ & $\mathrm{Y}$ & $\mathrm{R}$ \\
\hline 51 & 44.20 & 42.93 & 18.92 & 2.29 & 3.94 & 990.60 & 305.02 & 434.37 & 131.28 & 203.255 & $\mathrm{C}$ & $\mathrm{Y}$ & $\mathrm{R}$ \\
\hline 52 & 43.76 & 42.49 & 18.71 & 1.85 & 4.15 & 2156.46 & 304.95 & 421.39 & 40.27 & 203.255 & $\mathrm{C}$ & $\mathrm{Y}$ & $\mathrm{R}$ \\
\hline 53 & 43.76 & 42.49 & 18.71 & 1.85 & 4.15 & 2044.70 & 304.95 & 421.39 & 39.16 & 203.255 & $\mathrm{C}$ & $\mathrm{Y}$ & $\mathrm{R}$ \\
\hline 54 & 43.76 & 42.49 & 18.71 & 1.85 & 4.15 & 2044.70 & 304.95 & 421.39 & 35.60 & 203.255 & $\mathrm{C}$ & $\mathrm{Y}$ & $\mathrm{R}$ \\
\hline 55 & 43.76 & 42.49 & 18.71 & 1.85 & 4.15 & 1295.40 & 304.95 & 421.39 & 68.98 & 203.255 & $\mathrm{C}$ & $\mathrm{Y}$ & $\mathrm{R}$ \\
\hline 56 & 43.76 & 42.49 & 18.71 & 1.85 & 4.15 & 1295.40 & 304.95 & 421.39 & 71.20 & 203.255 & $\mathrm{C}$ & $\mathrm{Y}$ & $\mathrm{R}$ \\
\hline 57 & 43.76 & 42.49 & 18.71 & 1.85 & 4.15 & 990.60 & 304.95 & 421.39 & 80.10 & 203.255 & $\mathrm{C}$ & $\mathrm{Y}$ & $\mathrm{R}$ \\
\hline 58 & 43.76 & 42.49 & 18.71 & 1.85 & 4.15 & 685.80 & 304.95 & 421.39 & 86.78 & 203.255 & $\mathrm{C}$ & $\mathrm{Y}$ & $\mathrm{R}$ \\
\hline
\end{tabular}


Table 4: Continued.

\begin{tabular}{|c|c|c|c|c|c|c|c|c|c|c|c|c|c|}
\hline $\begin{array}{l}\text { S. } \\
\text { No. }\end{array}$ & $\begin{array}{c}W \\
(\mathrm{~mm})\end{array}$ & $\begin{array}{c}F \\
(\mathrm{~mm})\end{array}$ & $\begin{array}{c}L \\
(\mathrm{~mm})\end{array}$ & $\begin{array}{c}T \\
(\mathrm{~mm})\end{array}$ & $\begin{array}{c}R \\
(\mathrm{~mm})\end{array}$ & $\begin{array}{c}\text { Length } \\
(\mathrm{mm})\end{array}$ & $\begin{array}{c}F_{\mathrm{y}}(\mathrm{N} / \\
\left.\mathrm{mm}^{2}\right)\end{array}$ & $\begin{array}{c}F_{\mathrm{u}}(\mathrm{N} / \\
\left.\mathrm{mm}^{2}\right)\end{array}$ & $\begin{array}{l}P_{\text {Test }} \\
(\mathrm{kN})\end{array}$ & $\begin{array}{l}E(\mathrm{~N} / \\
\left.\mathrm{mm}^{2}\right)\end{array}$ & $\begin{array}{l}\text { Type of } \\
\text { comp. } \\
\text { member }\end{array}$ & $\begin{array}{l}\text { Torsion } \\
\text { allowed }\end{array}$ & $\begin{array}{c}\text { Forming } \\
\text { method }\end{array}$ \\
\hline 59 & 44.20 & 42.93 & 18.92 & 2.29 & 3.94 & 2540.00 & 330.10 & 429.05 & 34.27 & 203.255 & $\mathrm{C}$ & $\mathrm{Y}$ & $\mathrm{P}$ \\
\hline 60 & 44.20 & 42.93 & 18.92 & 2.29 & 3.94 & 2082.80 & 330.10 & 429.05 & 44.28 & 203.255 & $\mathrm{C}$ & $\mathrm{Y}$ & $\mathrm{P}$ \\
\hline 61 & 44.20 & 42.93 & 18.92 & 2.29 & 3.94 & 1600.20 & 330.10 & 429.05 & 70.53 & 203.255 & $\mathrm{C}$ & $\mathrm{Y}$ & $\mathrm{P}$ \\
\hline 62 & 44.20 & 42.93 & 18.92 & 2.29 & 3.94 & 1295.40 & 330.10 & 429.05 & 96.12 & 203.255 & $\mathrm{C}$ & $\mathrm{Y}$ & $\mathrm{P}$ \\
\hline 63 & 44.20 & 42.93 & 18.92 & 2.29 & 3.94 & 990.60 & 330.10 & 429.05 & 117.48 & 203.255 & $\mathrm{C}$ & Y & $\mathrm{P}$ \\
\hline 64 & 43.76 & 42.49 & 18.71 & 1.85 & 4.15 & 2260.60 & 308.33 & 413.90 & 36.49 & 203.255 & $\mathrm{C}$ & $\mathrm{Y}$ & $\mathrm{P}$ \\
\hline 65 & 43.76 & 42.49 & 18.71 & 1.85 & 4.15 & 1981.20 & 308.33 & 413.90 & 46.73 & 203.255 & $\mathrm{C}$ & $\mathrm{Y}$ & $\mathrm{P}$ \\
\hline 66 & 43.76 & 42.49 & 18.71 & 1.85 & 4.15 & 1752.60 & 308.33 & 413.90 & 49.84 & 203.255 & $\mathrm{C}$ & $\mathrm{Y}$ & $\mathrm{P}$ \\
\hline 67 & 43.76 & 42.49 & 18.71 & 1.85 & 4.15 & 1447.80 & 308.33 & 413.90 & 62.08 & 203.255 & $\mathrm{C}$ & $\mathrm{Y}$ & $\mathrm{P}$ \\
\hline 68 & 43.76 & 42.49 & 18.71 & 1.85 & 4.15 & 990.60 & 308.33 & 413.90 & 85.89 & 203.255 & $\mathrm{C}$ & $\mathrm{Y}$ & $\mathrm{P}$ \\
\hline 69 & 43.76 & 42.49 & 18.71 & 1.85 & 4.15 & 685.80 & 308.33 & 413.90 & 89.89 & 203.255 & $\mathrm{C}$ & $\mathrm{Y}$ & $\mathrm{P}$ \\
\hline 70 & 156.57 & 81.10 & 18.11 & 1.14 & 2.78 & 1600.20 & 223.30 & 319.90 & 43.61 & 203.255 & $\mathrm{C}$ & $\mathrm{Y}$ & $\mathrm{P}$ \\
\hline 71 & 155.98 & 81.08 & 17.04 & 1.14 & 2.72 & 1905.51 & 220.14 & 317.22 & 46.28 & 203.255 & $\mathrm{C}$ & $\mathrm{Y}$ & $\mathrm{P}$ \\
\hline 72 & 156.97 & 81.08 & 17.04 & 1.16 & 2.76 & 3075.94 & 220.14 & 317.22 & 36.49 & 203.255 & $\mathrm{C}$ & $\mathrm{Y}$ & $\mathrm{P}$ \\
\hline 73 & 155.37 & 81.20 & 17.63 & 1.15 & 2.81 & 3073.40 & 220.14 & 317.22 & 37.38 & 203.255 & $\mathrm{C}$ & $\mathrm{Y}$ & $\mathrm{P}$ \\
\hline 74 & 156.57 & 80.49 & 18.29 & 1.22 & 2.95 & 1905.00 & 223.72 & 310.81 & 52.51 & 203.255 & $\mathrm{C}$ & $\mathrm{Y}$ & $\mathrm{P}$ \\
\hline 75 & 231.57 & 81.25 & 17.27 & 1.14 & 2.72 & 1829.05 & 224.55 & 321.49 & 42.72 & 203.255 & $\mathrm{C}$ & $\mathrm{Y}$ & $\mathrm{P}$ \\
\hline 76 & 231.98 & 81.13 & 17.48 & 1.14 & 2.82 & 2416.05 & 223.30 & 319.90 & 38.94 & 203.255 & $\mathrm{C}$ & $\mathrm{Y}$ & $\mathrm{P}$ \\
\hline 77 & 232.18 & 81.13 & 17.37 & 1.12 & 2.69 & 2997.20 & 223.30 & 319.90 & 33.82 & 203.255 & $\mathrm{C}$ & $\mathrm{Y}$ & $\mathrm{P}$ \\
\hline 78 & 230.58 & 81.25 & 18.44 & 1.23 & 2.94 & 2413.76 & 227.78 & 306.95 & 48.06 & 203.255 & $\mathrm{C}$ & $\mathrm{Y}$ & $\mathrm{P}$ \\
\hline 79 & 222.05 & 114.20 & 19.94 & 1.21 & 2.90 & 1906.78 & 219.24 & 307.91 & 54.74 & 203.255 & $\mathrm{C}$ & $\mathrm{Y}$ & $\mathrm{P}$ \\
\hline 80 & 222.83 & 113.69 & 19.46 & 1.21 & 3.00 & 2516.38 & 244.04 & 319.63 & 53.85 & 203.255 & $\mathrm{C}$ & $\mathrm{Y}$ & $\mathrm{P}$ \\
\hline 81 & 222.66 & 114.00 & 19.13 & 1.22 & 2.99 & 2518.66 & 233.23 & 311.22 & 52.51 & 203.255 & $\mathrm{C}$ & $\mathrm{Y}$ & $\mathrm{P}$ \\
\hline 82 & 153.95 & 62.79 & 25.37 & 0.80 & 0.00 & 1905.00 & 242.00 & 360.00 & 21.80 & 201.000 & $\mathrm{C}$ & $\mathrm{Y}$ & $\mathrm{P}$ \\
\hline 83 & 153.95 & 62.89 & 25.60 & 0.81 & 0.00 & 1600.20 & 242.00 & 360.00 & 23.04 & 201.000 & $\mathrm{C}$ & $\mathrm{Y}$ & $\mathrm{P}$ \\
\hline 84 & 152.02 & 62.97 & 25.57 & 0.80 & 0.00 & 1295.40 & 242.00 & 360.00 & 23.62 & 201.000 & $\mathrm{C}$ & $\mathrm{Y}$ & $\mathrm{P}$ \\
\hline 85 & 152.30 & 49.28 & 17.74 & 1.64 & 0.00 & 1905.00 & 233.00 & 360.00 & 65.83 & 208.000 & $\mathrm{C}$ & $\mathrm{Y}$ & $\mathrm{P}$ \\
\hline 86 & 178.23 & 61.85 & 24.62 & 1.63 & 0.00 & 1905.00 & 233.00 & 360.00 & 75.39 & 208.000 & $\mathrm{C}$ & $\mathrm{Y}$ & $\mathrm{P}$ \\
\hline 87 & 177.93 & 61.62 & 24.77 & 1.64 & 0.00 & 1600.20 & 233.00 & 360.00 & 75.84 & 208.000 & $\mathrm{C}$ & $\mathrm{Y}$ & $\mathrm{P}$ \\
\hline 88 & 176.73 & 61.98 & 25.71 & 1.64 & 0.00 & 1295.40 & 233.00 & 360.00 & 80.29 & 208.000 & $\mathrm{C}$ & $\mathrm{Y}$ & $\mathrm{P}$ \\
\hline 89 & 90.00 & 50.00 & 20.00 & 3.15 & 3.15 & 1915.00 & 350.00 & 471.00 & 119.00 & 219.800 & $\mathrm{C}$ & $\mathrm{Y}$ & $\mathrm{P}$ \\
\hline 90 & 90.00 & 50.00 & 20.00 & 3.15 & 3.15 & 1915.00 & 350.00 & 471.00 & 118.00 & 219.800 & $\mathrm{C}$ & $\mathrm{Y}$ & $\mathrm{P}$ \\
\hline 91 & 90.00 & 50.00 & 20.00 & 3.15 & 3.15 & 1915.00 & 350.00 & 471.00 & 113.00 & 219.800 & $\mathrm{C}$ & $\mathrm{Y}$ & $\mathrm{P}$ \\
\hline 92 & 92.00 & 37.00 & 12.00 & 1.91 & 2.40 & 276.00 & 358.00 & 450.00 & 192.90 & 203.255 & $\mathrm{C}$ & $\mathrm{Y}$ & $\mathrm{R}$ \\
\hline 93 & 151.00 & 34.00 & 8.00 & 0.93 & 2.40 & 453.00 & 309.00 & 360.00 & 38.20 & 203.255 & $\mathrm{C}$ & $\mathrm{Y}$ & $\mathrm{R}$ \\
\hline 94 & 92.00 & 37.00 & 11.00 & 2.14 & 2.40 & 276.00 & 358.00 & 450.00 & 193.60 & 203.255 & $\mathrm{C}$ & $\mathrm{Y}$ & $\mathrm{R}$ \\
\hline 95 & 92.00 & 37.00 & 12.00 & 2.15 & 2.40 & 276.00 & 358.00 & 450.00 & 194.60 & 203.255 & $\mathrm{C}$ & $\mathrm{Y}$ & $\mathrm{R}$ \\
\hline 96 & 92.00 & 37.00 & 11.00 & 2.14 & 2.40 & 276.00 & 358.00 & 450.00 & 213.60 & 203.255 & $\mathrm{C}$ & $\mathrm{Y}$ & $\mathrm{R}$ \\
\hline 97 & 152.00 & 35.00 & 8.00 & 0.89 & 2.40 & 456.00 & 309.00 & 360.00 & 43.30 & 203.255 & $\mathrm{C}$ & $\mathrm{Y}$ & $\mathrm{R}$ \\
\hline 98 & 152.00 & 35.00 & 8.00 & 0.87 & 2.40 & 456.00 & 309.00 & 360.00 & 42.60 & 203.255 & $\mathrm{C}$ & $\mathrm{Y}$ & $\mathrm{R}$ \\
\hline 99 & 152.00 & 34.00 & 8.00 & 0.91 & 2.40 & 456.00 & 309.00 & 360.00 & 43.30 & 203.255 & $\mathrm{C}$ & $\mathrm{Y}$ & $\mathrm{R}$ \\
\hline 100 & 92.00 & 37.00 & 12.00 & 1.92 & 2.40 & 276.00 & 366.00 & 450.00 & 165.40 & 203.255 & $\mathrm{C}$ & $\mathrm{Y}$ & $\mathrm{R}$ \\
\hline 101 & 92.00 & 36.00 & 12.00 & 1.91 & 2.40 & 276.00 & 366.00 & 450.00 & 184.00 & 203.255 & $\mathrm{C}$ & $\mathrm{Y}$ & $\mathrm{R}$ \\
\hline 102 & 92.00 & 37.00 & 12.00 & 1.91 & 2.40 & 276.00 & 366.00 & 450.00 & 182.90 & 203.255 & $\mathrm{C}$ & $\mathrm{Y}$ & $\mathrm{R}$ \\
\hline 103 & 92.00 & 37.00 & 12.00 & 1.92 & 2.40 & 276.00 & 366.00 & 450.00 & 158.80 & 203.255 & $\mathrm{C}$ & $\mathrm{Y}$ & $\mathrm{R}$ \\
\hline 104 & 92.00 & 37.00 & 12.00 & 1.91 & 2.40 & 276.00 & 366.00 & 450.00 & 182.60 & 203.255 & $\mathrm{C}$ & $\mathrm{Y}$ & $\mathrm{R}$ \\
\hline 105 & 152.00 & 35.00 & 8.00 & 0.90 & 2.40 & 456.00 & 302.00 & 360.00 & 40.20 & 203.255 & $\mathrm{C}$ & $\mathrm{Y}$ & $\mathrm{R}$ \\
\hline 106 & 152.00 & 35.00 & 8.00 & 0.89 & 2.40 & 456.00 & 302.00 & 360.00 & 39.60 & 203.255 & $\mathrm{C}$ & $\mathrm{Y}$ & $\mathrm{R}$ \\
\hline 107 & 152.00 & 35.00 & 8.00 & 0.89 & 2.40 & 456.00 & 302.00 & 360.00 & 37.00 & 203.255 & $\mathrm{C}$ & $\mathrm{Y}$ & $\mathrm{R}$ \\
\hline 108 & 152.00 & 35.00 & 8.00 & 0.89 & 2.40 & 456.00 & 302.00 & 360.00 & 38.20 & 203.255 & $\mathrm{C}$ & $\mathrm{Y}$ & $\mathrm{R}$ \\
\hline 109 & 152.00 & 35.00 & 8.00 & 0.89 & 2.40 & 456.00 & 302.00 & 360.00 & 41.00 & 203.255 & $\mathrm{C}$ & $\mathrm{Y}$ & $\mathrm{R}$ \\
\hline 110 & 85.80 & 56.00 & 15.00 & 1.90 & 2.90 & 1080.00 & 213.00 & 360.00 & 66.71 & 210.000 & $\mathrm{C}$ & $\mathrm{N}$ & $\mathrm{P}$ \\
\hline 111 & 85.60 & 57.20 & 16.40 & 1.90 & 2.90 & 1080.00 & 213.00 & 360.00 & 67.69 & 210.000 & $\mathrm{C}$ & $\mathrm{N}$ & $\mathrm{P}$ \\
\hline 112 & 86.20 & 55.40 & 17.00 & 1.90 & 2.90 & 2000.00 & 213.00 & 360.00 & 51.99 & 210.000 & $\mathrm{C}$ & $\mathrm{N}$ & $\mathrm{P}$ \\
\hline 113 & 87.20 & 55.50 & 17.00 & 1.75 & 2.60 & 2000.00 & 213.00 & 360.00 & 49.05 & 210.000 & $\mathrm{C}$ & $\mathrm{N}$ & $\mathrm{P}$ \\
\hline 114 & 96.00 & 65.50 & 20.00 & 1.75 & 2.60 & 1480.00 & 213.00 & 360.00 & 69.65 & 210.000 & $\mathrm{C}$ & $\mathrm{N}$ & $\mathrm{P}$ \\
\hline 115 & 94.00 & 65.80 & 20.00 & 1.75 & 2.60 & 1480.00 & 213.00 & 360.00 & 71.61 & 210.000 & $\mathrm{C}$ & $\mathrm{N}$ & $\mathrm{P}$ \\
\hline 116 & 99.80 & 64.50 & 19.80 & 1.85 & 2.80 & 2000.00 & 213.00 & 360.00 & 64.75 & 210.000 & $\mathrm{C}$ & $\mathrm{N}$ & $\mathrm{P}$ \\
\hline
\end{tabular}


TABle 4: Continued.

\begin{tabular}{|c|c|c|c|c|c|c|c|c|c|c|c|c|c|}
\hline $\begin{array}{l}\text { S. } \\
\text { No. }\end{array}$ & $\begin{array}{c}W \\
(\mathrm{~mm})\end{array}$ & $\begin{array}{c}F \\
(\mathrm{~mm})\end{array}$ & $\begin{array}{c}L \\
(\mathrm{~mm})\end{array}$ & $\begin{array}{c}T \\
(\mathrm{~mm})\end{array}$ & $\begin{array}{c}R \\
(\mathrm{~mm})\end{array}$ & $\begin{array}{l}\text { Length } \\
(\mathrm{mm})\end{array}$ & $\begin{array}{c}F_{\mathrm{y}}(\mathrm{N} / \\
\left.\mathrm{mm}^{2}\right)\end{array}$ & $\begin{array}{c}F_{\mathrm{u}}(\mathrm{N} / \\
\left.\mathrm{mm}^{2}\right)\end{array}$ & $\begin{array}{l}P_{\text {Test }} \\
(\mathrm{kN})\end{array}$ & $\begin{array}{l}E(\mathrm{~N} / \\
\left.\mathrm{mm}^{2}\right)\end{array}$ & $\begin{array}{l}\text { Type of } \\
\text { comp. } \\
\text { member }\end{array}$ & $\begin{array}{l}\text { Torsion } \\
\text { allowed }\end{array}$ & $\begin{array}{c}\text { Forming } \\
\text { method }\end{array}$ \\
\hline 117 & 99.00 & 66.50 & 20.50 & 1.90 & 2.90 & 2000.00 & 213.00 & 360.00 & 69.65 & 210.000 & $\mathrm{C}$ & $\mathrm{N}$ & $\mathrm{P}$ \\
\hline 118 & 96.00 & 72.00 & 21.20 & 3.00 & 4.50 & 1120.00 & 211.00 & 360.00 & 156.96 & 210.000 & $\mathrm{C}$ & $\mathrm{N}$ & $\mathrm{P}$ \\
\hline 119 & 97.70 & 70.30 & 22.10 & 3.00 & 4.50 & 2000.00 & 211.00 & 360.00 & 123.61 & 210.000 & $\mathrm{C}$ & $\mathrm{N}$ & $\mathrm{P}$ \\
\hline 120 & 97.50 & 70.00 & 21.60 & 3.00 & 4.50 & 2000.00 & 211.00 & 360.00 & 130.47 & 210.000 & $\mathrm{C}$ & $\mathrm{N}$ & $\mathrm{P}$ \\
\hline 121 & 136.50 & 55.50 & 24.50 & 3.00 & 4.50 & 1700.00 & 211.00 & 360.00 & 186.39 & 210.000 & $\mathrm{C}$ & $\mathrm{N}$ & $\mathrm{P}$ \\
\hline 122 & 121.00 & 83.20 & 25.00 & 3.00 & 4.50 & 1700.00 & 211.00 & 360.00 & 167.75 & 210.000 & $\mathrm{C}$ & $\mathrm{N}$ & $\mathrm{P}$ \\
\hline 123 & 121.80 & 82.70 & 25.20 & 3.00 & 4.50 & 1700.00 & 211.00 & 360.00 & 150.09 & 210.000 & $\mathrm{C}$ & $\mathrm{N}$ & $\mathrm{P}$ \\
\hline 124 & 119.30 & 85.50 & 25.10 & 3.00 & 4.50 & 2000.00 & 211.00 & 360.00 & 143.23 & 210.000 & $\mathrm{C}$ & $\mathrm{N}$ & $\mathrm{P}$ \\
\hline 125 & 121.30 & 84.60 & 25.60 & 3.00 & 4.50 & 2000.00 & 211.00 & 360.00 & 154.02 & 210.000 & $\mathrm{C}$ & $\mathrm{N}$ & $\mathrm{P}$ \\
\hline 126 & 121.00 & 86.10 & 25.50 & 1.90 & 2.90 & 2000.00 & 213.00 & 360.00 & 94.18 & 210.000 & $\mathrm{C}$ & $\mathrm{N}$ & $\mathrm{P}$ \\
\hline 127 & 121.00 & 86.10 & 25.50 & 1.90 & 2.90 & 2000.00 & 213.00 & 360.00 & 96.14 & 210.000 & $\mathrm{C}$ & $\mathrm{N}$ & $\mathrm{P}$ \\
\hline 128 & 82.50 & 57.00 & 16.00 & 1.80 & 2.70 & 295.00 & 213.00 & 360.00 & 77.50 & 210.000 & SC & $\mathrm{N}$ & $\mathrm{P}$ \\
\hline 129 & 82.00 & 57.00 & 17.00 & 1.80 & 2.70 & 296.00 & 213.00 & 360.00 & 77.50 & 210.000 & SC & $\mathrm{N}$ & $\mathrm{P}$ \\
\hline 130 & 97.00 & 66.00 & 21.00 & 1.85 & 2.80 & 350.00 & 213.00 & 360.00 & 87.31 & 210.000 & SC & $\mathrm{N}$ & $\mathrm{P}$ \\
\hline 131 & 95.00 & 67.00 & 21.00 & 1.90 & 2.90 & 350.00 & 213.00 & 360.00 & 96.14 & 210.000 & SC & $\mathrm{N}$ & $\mathrm{P}$ \\
\hline 132 & 97.00 & 70.00 & 23.00 & 3.00 & 4.50 & 348.00 & 211.00 & 360.00 & 200.12 & 210.000 & SC & $\mathrm{N}$ & $\mathrm{P}$ \\
\hline 133 & 97.00 & 70.00 & 23.00 & 3.00 & 4.50 & 348.00 & 211.00 & 360.00 & 192.28 & 210.000 & SC & $\mathrm{N}$ & $\mathrm{P}$ \\
\hline 134 & 114.00 & 84.00 & 27.00 & 3.10 & 4.70 & 397.00 & 211.00 & 360.00 & 214.84 & 210.000 & SC & $\mathrm{N}$ & $\mathrm{P}$ \\
\hline 135 & 115.00 & 88.00 & 26.00 & 3.10 & 4.70 & 399.00 & 211.00 & 360.00 & 213.86 & 210.000 & SC & $\mathrm{N}$ & $\mathrm{P}$ \\
\hline 136 & 121.00 & 88.00 & 26.00 & 3.10 & 4.70 & 400.00 & 211.00 & 360.00 & 208.95 & 210.000 & SC & $\mathrm{N}$ & $\mathrm{P}$ \\
\hline 137 & 120.00 & 87.00 & 26.00 & 1.90 & 2.90 & 400.00 & 213.00 & 360.00 & 119.68 & 210.000 & SC & $\mathrm{N}$ & $\mathrm{P}$ \\
\hline 138 & 123.00 & 86.00 & 25.00 & 1.90 & 2.90 & 400.00 & 213.00 & 360.00 & 117.72 & 210.000 & SC & $\mathrm{N}$ & $\mathrm{P}$ \\
\hline 139 & 85.70 & 34.90 & 9.50 & 1.60 & 3.20 & 200.00 & 340.60 & 443.60 & 84.55 & 205.000 & SC & $\mathrm{Y}$ & $\mathrm{R}$ \\
\hline 140 & 85.70 & 34.90 & 9.50 & 1.60 & 3.20 & 200.00 & 340.60 & 443.60 & 84.70 & 205.000 & SC & $\mathrm{Y}$ & $\mathrm{R}$ \\
\hline 141 & 85.70 & 34.90 & 9.50 & 1.60 & 3.20 & 200.00 & 340.60 & 443.60 & 86.75 & 205.000 & SC & $\mathrm{Y}$ & $\mathrm{R}$ \\
\hline 142 & 147.28 & 36.18 & 10.09 & 1.29 & 2.58 & 265.00 & 262.20 & 336.00 & 54.30 & 210.000 & $\mathrm{C}$ & $\mathrm{Y}$ & $\mathrm{R}$ \\
\hline 143 & 147.28 & 36.18 & 10.09 & 1.29 & 2.58 & 265.00 & 262.20 & 336.00 & 54.40 & 210.000 & $\mathrm{C}$ & $\mathrm{Y}$ & $\mathrm{R}$ \\
\hline 144 & 147.28 & 36.18 & 10.09 & 1.29 & 2.58 & 265.00 & 262.20 & 336.00 & 53.25 & 210.000 & $\mathrm{C}$ & $\mathrm{Y}$ & $\mathrm{R}$ \\
\hline 145 & 300.46 & 101.56 & 20.23 & 0.63 & 1.50 & 2690.00 & 391.00 & 482.00 & 16.00 & 216.000 & $\mathrm{C}$ & $\mathrm{N}$ & $\mathrm{R}$ \\
\hline 146 & 300.98 & 101.98 & 21.24 & 0.64 & 1.50 & 2690.00 & 395.00 & 490.00 & 16.20 & 216.000 & $\mathrm{C}$ & $\mathrm{N}$ & $\mathrm{R}$ \\
\hline 147 & 300.48 & 101.78 & 20.64 & 0.64 & 1.50 & 2690.00 & 398.00 & 483.00 & 15.50 & 216.000 & $\mathrm{C}$ & $\mathrm{N}$ & $\mathrm{R}$ \\
\hline 148 & 301.30 & 101.50 & 20.85 & 0.65 & 1.50 & 2690.00 & 288.00 & 384.00 & 14.50 & 216.000 & $\mathrm{C}$ & $\mathrm{N}$ & $\mathrm{R}$ \\
\hline 149 & 301.68 & 102.38 & 21.14 & 0.94 & 1.50 & 2690.00 & 464.00 & 552.00 & 36.90 & 216.000 & $\mathrm{C}$ & $\mathrm{N}$ & $\mathrm{R}$ \\
\hline 150 & 301.58 & 102.38 & 20.94 & 0.94 & 1.50 & 2690.00 & 460.00 & 545.00 & 35.00 & 216.000 & $\mathrm{C}$ & $\mathrm{N}$ & $\mathrm{R}$ \\
\hline 151 & 301.18 & 102.48 & 20.54 & 0.94 & 1.50 & 2690.00 & 460.00 & 546.00 & 37.10 & 216.000 & $\mathrm{C}$ & $\mathrm{N}$ & $\mathrm{R}$ \\
\hline 152 & 300.22 & 101.52 & 20.46 & 0.96 & 1.50 & 2690.00 & 475.00 & 549.00 & 34.50 & 216.000 & $\mathrm{C}$ & $\mathrm{N}$ & $\mathrm{R}$ \\
\hline 153 & 302.20 & 102.90 & 21.75 & 1.45 & 1.50 & 2690.00 & 382.00 & 463.00 & 76.60 & 216.000 & $\mathrm{C}$ & $\mathrm{N}$ & $\mathrm{R}$ \\
\hline 154 & 302.96 & 102.86 & 21.63 & 1.43 & 1.50 & 2690.00 & 379.00 & 466.00 & 70.00 & 216.000 & $\mathrm{C}$ & $\mathrm{N}$ & $\mathrm{R}$ \\
\hline 155 & 302.56 & 102.56 & 22.18 & 1.38 & 1.50 & 2690.00 & 395.00 & 467.00 & 71.30 & 216.000 & $\mathrm{C}$ & $\mathrm{N}$ & $\mathrm{R}$ \\
\hline 156 & 303.38 & 103.18 & 24.79 & 1.39 & 1.50 & 2690.00 & 393.00 & 478.00 & 73.00 & 216.000 & $\mathrm{C}$ & $\mathrm{N}$ & $\mathrm{R}$ \\
\hline 157 & 302.00 & 103.60 & 25.00 & 1.40 & 1.50 & 2690.00 & 380.00 & 470.00 & 57.00 & 216.000 & $\mathrm{C}$ & $\mathrm{N}$ & $\mathrm{R}$ \\
\hline 158 & 302.18 & 102.58 & 22.49 & 1.39 & 1.50 & 2690.00 & 381.00 & 474.00 & 69.00 & 216.000 & $\mathrm{C}$ & $\mathrm{N}$ & $\mathrm{R}$ \\
\hline 159 & 82.60 & 41.63 & 9.86 & 1.22 & 2.59 & 303.53 & 225.92 & 300.27 & 46.28 & 203.255 & $\mathrm{C}$ & $\mathrm{Y}$ & $\mathrm{P}$ \\
\hline 160 & 116.69 & 41.50 & 9.88 & 1.22 & 2.64 & 304.29 & 225.92 & 300.27 & 44.72 & 203.255 & $\mathrm{C}$ & $\mathrm{Y}$ & $\mathrm{P}$ \\
\hline 161 & 154.18 & 42.06 & 9.65 & 1.22 & 3.09 & 278.38 & 225.92 & 300.27 & 45.17 & 203.255 & SC & $\mathrm{Y}$ & $\mathrm{P}$ \\
\hline 162 & 79.86 & 81.71 & 17.22 & 1.20 & 1.87 & 457.71 & 230.06 & 315.01 & 58.74 & 203.255 & SC & $\mathrm{Y}$ & $\mathrm{P}$ \\
\hline 163 & 79.40 & 81.58 & 17.27 & 1.21 & 1.76 & 457.71 & 230.06 & 315.01 & 60.52 & 203.255 & SC & $\mathrm{Y}$ & $\mathrm{P}$ \\
\hline 164 & 151.41 & 81.94 & 17.30 & 1.20 & 1.97 & 456.69 & 230.06 & 315.01 & 57.85 & 203.255 & SC & $\mathrm{Y}$ & $\mathrm{P}$ \\
\hline 165 & 150.83 & 82.25 & 16.92 & 1.20 & 1.93 & 456.69 & 230.06 & 315.01 & 60.52 & 203.255 & SC & $\mathrm{Y}$ & $\mathrm{P}$ \\
\hline 166 & 228.19 & 81.64 & 17.50 & 1.20 & 1.87 & 559.31 & 230.06 & 315.01 & 56.96 & 203.255 & SC & $\mathrm{Y}$ & $\mathrm{P}$ \\
\hline 167 & 227.81 & 81.86 & 17.55 & 1.19 & 2.14 & 685.04 & 230.06 & 315.01 & 56.96 & 203.255 & $\mathrm{C}$ & $\mathrm{Y}$ & $\mathrm{P}$ \\
\hline 168 & 299.97 & 81.86 & 17.30 & 1.19 & 1.98 & 558.80 & 230.06 & 315.01 & 56.96 & 203.255 & SC & $\mathrm{Y}$ & $\mathrm{P}$ \\
\hline 169 & 300.74 & 82.04 & 17.63 & 1.19 & 2.41 & 913.64 & 238.60 & 321.42 & 53.40 & 203.255 & $\mathrm{C}$ & $\mathrm{Y}$ & $\mathrm{P}$ \\
\hline 170 & 299.72 & 81.79 & 17.32 & 1.20 & 1.98 & 558.04 & 230.06 & 315.01 & 56.07 & 203.255 & SC & $\mathrm{Y}$ & $\mathrm{P}$ \\
\hline 171 & 80.72 & 114.10 & 19.13 & 1.14 & 2.52 & 647.70 & 223.30 & 319.90 & 51.18 & 203.255 & SC & $\mathrm{Y}$ & $\mathrm{P}$ \\
\hline 172 & 80.16 & 114.20 & 19.20 & 1.14 & 2.67 & 647.19 & 223.30 & 319.90 & 52.51 & 203.255 & SC & $\mathrm{Y}$ & $\mathrm{P}$ \\
\hline 173 & 114.88 & 114.10 & 19.23 & 1.13 & 2.65 & 646.94 & 224.55 & 321.49 & 52.96 & 203.255 & SC & $\mathrm{Y}$ & $\mathrm{P}$ \\
\hline 174 & 114.50 & 114.00 & 19.25 & 1.14 & 2.57 & 647.19 & 224.55 & 321.49 & 53.40 & 203.255 & SC & $\mathrm{Y}$ & $\mathrm{P}$ \\
\hline
\end{tabular}


TABle 4: Continued.

\begin{tabular}{|c|c|c|c|c|c|c|c|c|c|c|c|c|c|}
\hline $\begin{array}{l}\text { S. } \\
\text { No. }\end{array}$ & $\begin{array}{c}W \\
(\mathrm{~mm})\end{array}$ & $\begin{array}{c}F \\
(\mathrm{~mm})\end{array}$ & $\begin{array}{c}L \\
(\mathrm{~mm})\end{array}$ & $\begin{array}{c}T \\
(\mathrm{~mm})\end{array}$ & $\begin{array}{c}R \\
(\mathrm{~mm})\end{array}$ & $\begin{array}{l}\text { Length } \\
(\mathrm{mm})\end{array}$ & $\begin{array}{c}F_{\mathrm{y}}(\mathrm{N} / \\
\left.\mathrm{mm}^{2}\right)\end{array}$ & $\begin{array}{c}F_{\mathrm{u}}(\mathrm{N} / \\
\left.\mathrm{mm}^{2}\right)\end{array}$ & $\begin{array}{l}P_{\text {Test }} \\
(\mathrm{kN})\end{array}$ & $\begin{array}{l}E(\mathrm{~N} / \\
\left.\mathrm{mm}^{2}\right)\end{array}$ & $\begin{array}{l}\text { Type of } \\
\text { comp. } \\
\text { member }\end{array}$ & $\begin{array}{l}\text { Torsion } \\
\text { allowed }\end{array}$ & $\begin{array}{c}\text { Forming } \\
\text { method }\end{array}$ \\
\hline 175 & 281.18 & 145.36 & 34.21 & 1.55 & 2.20 & 891.29 & 412.02 & 546.72 & 138.62 & 203.255 & SC & $\mathrm{Y}$ & $\mathrm{P}$ \\
\hline 176 & 281.43 & 146.15 & 33.40 & 1.55 & 2.41 & 891.54 & 412.02 & 546.72 & 139.73 & 203.255 & SC & $\mathrm{Y}$ & $\mathrm{P}$ \\
\hline 177 & 224.23 & 113.79 & 19.35 & 1.22 & 2.94 & 646.94 & 368.06 & 490.09 & 67.64 & 203.255 & SC & $\mathrm{Y}$ & $\mathrm{P}$ \\
\hline 178 & 223.65 & 115.09 & 18.95 & 1.28 & 2.94 & 647.70 & 198.64 & 290.07 & 61.41 & 203.255 & SC & $\mathrm{Y}$ & $\mathrm{P}$ \\
\hline 179 & 223.24 & 114.88 & 19.51 & 1.29 & 3.08 & 647.95 & 198.64 & 290.07 & 64.97 & 203.255 & SC & $\mathrm{Y}$ & $\mathrm{P}$ \\
\hline 180 & 331.72 & 114.88 & 19.05 & 1.27 & 2.94 & 762.00 & 198.64 & 290.07 & 60.52 & 203.255 & SC & $\mathrm{Y}$ & $\mathrm{P}$ \\
\hline 181 & 330.71 & 115.39 & 19.05 & 1.28 & 2.94 & 971.30 & 203.19 & 291.38 & 62.30 & 203.255 & $\mathrm{C}$ & $\mathrm{Y}$ & $\mathrm{P}$ \\
\hline 182 & 440.94 & 113.69 & 19.18 & 1.24 & 2.77 & 761.75 & 201.19 & 290.41 & 55.63 & 203.255 & $\mathrm{C}$ & $\mathrm{Y}$ & $\mathrm{P}$ \\
\hline 183 & 441.45 & 113.69 & 19.10 & 1.25 & 2.56 & 1295.15 & 202.08 & 291.24 & 49.84 & 203.255 & $\mathrm{C}$ & $\mathrm{Y}$ & $\mathrm{P}$ \\
\hline 184 & 114.71 & 113.79 & 19.76 & 1.23 & 2.99 & 2518.66 & 236.60 & 313.56 & 48.95 & 203.255 & C & $\mathrm{Y}$ & $\mathrm{P}$ \\
\hline 185 & 152.40 & 50.80 & 12.70 & 1.22 & 1.22 & 457.20 & 293.24 & 449.99 & 55.18 & 203.255 & C & $\mathrm{Y}$ & $\mathrm{P}$ \\
\hline 186 & 146.05 & 63.50 & 25.40 & 2.95 & 2.95 & 438.15 & 274.70 & 360.00 & 231.40 & 203.255 & SC & $\mathrm{Y}$ & $\mathrm{P}$ \\
\hline 187 & 127.00 & 63.50 & 12.70 & 2.03 & 2.03 & 381.00 & 293.24 & 449.99 & 113.48 & 203.255 & SC & $\mathrm{Y}$ & $\mathrm{P}$ \\
\hline 188 & 101.60 & 57.15 & 9.53 & 2.03 & 2.03 & 304.80 & 245.42 & 360.00 & 106.36 & 203.255 & SC & $\mathrm{Y}$ & $\mathrm{P}$ \\
\hline 189 & 88.90 & 50.80 & 9.53 & 2.03 & 2.03 & 266.70 & 304.06 & 449.99 & 109.47 & 203.255 & SC & $\mathrm{Y}$ & $\mathrm{P}$ \\
\hline 190 & 101.60 & 44.45 & 15.88 & 3.25 & 3.25 & 304.80 & 317.90 & 449.99 & 226.06 & 203.255 & SC & $\mathrm{Y}$ & $\mathrm{P}$ \\
\hline 191 & 76.20 & 38.10 & 9.53 & 3.25 & 3.25 & 228.60 & 324.11 & 449.99 & 159.76 & 203.255 & SC & $\mathrm{Y}$ & $\mathrm{P}$ \\
\hline 192 & 69.85 & 25.40 & 12.70 & 1.63 & 1.63 & 209.55 & 327.21 & 449.99 & 64.53 & 203.255 & $\mathrm{C}$ & $\mathrm{Y}$ & $\mathrm{P}$ \\
\hline 193 & 69.85 & 25.40 & 12.70 & 2.03 & 2.03 & 209.55 & 330.31 & 449.99 & 86.33 & 203.255 & $\mathrm{C}$ & $\mathrm{Y}$ & $\mathrm{P}$ \\
\hline 194 & 47.63 & 22.23 & 6.35 & 1.22 & 1.22 & 142.88 & 347.26 & 449.99 & 34.09 & 203.255 & SC & $\mathrm{Y}$ & $\mathrm{P}$ \\
\hline 195 & 44.45 & 25.40 & 6.35 & 1.22 & 1.22 & 133.35 & 376.61 & 449.99 & 41.03 & 203.255 & SC & $\mathrm{Y}$ & $\mathrm{P}$ \\
\hline 196 & 254.00 & 88.90 & 19.05 & 1.22 & 1.22 & 762.00 & 183.69 & 360.00 & 42.19 & 203.255 & $\mathrm{C}$ & $\mathrm{Y}$ & $\mathrm{P}$ \\
\hline 197 & 203.20 & 101.60 & 19.05 & 1.22 & 1.22 & 609.60 & 186.72 & 360.00 & 45.39 & 203.255 & SC & $\mathrm{Y}$ & $\mathrm{P}$ \\
\hline 198 & 152.40 & 101.60 & 19.05 & 1.22 & 1.22 & 457.20 & 191.40 & 360.00 & 41.21 & 203.255 & SC & $\mathrm{Y}$ & $\mathrm{P}$ \\
\hline 199 & 101.60 & 101.60 & 19.05 & 1.22 & 1.22 & 304.80 & 183.69 & 360.00 & 41.96 & 203.255 & SC & $\mathrm{Y}$ & $\mathrm{P}$ \\
\hline 200 & 50.80 & 50.80 & 9.53 & 1.22 & 1.22 & 152.40 & 192.92 & 360.00 & 32.31 & 203.255 & SC & $\mathrm{Y}$ & $\mathrm{P}$ \\
\hline 201 & 84.00 & 27.00 & 7.49 & 0.56 & 0.48 & 350.01 & 301.58 & 449.99 & 14.42 & 203.255 & $\mathrm{C}$ & $\mathrm{Y}$ & $\mathrm{P}$ \\
\hline 202 & 81.99 & 26.49 & 5.74 & 0.56 & 0.48 & 270.00 & 301.58 & 449.99 & 12.19 & 203.255 & $\mathrm{C}$ & $\mathrm{Y}$ & $\mathrm{P}$ \\
\hline 203 & 29.39 & 27.20 & 8.20 & 0.56 & 0.48 & 267.97 & 301.58 & 449.99 & 14.60 & 203.255 & $\mathrm{C}$ & $\mathrm{Y}$ & $\mathrm{P}$ \\
\hline 204 & 30.10 & 27.61 & 5.59 & 0.56 & 0.48 & 204.98 & 301.58 & 449.99 & 13.39 & 203.255 & $\mathrm{C}$ & $\mathrm{Y}$ & $\mathrm{P}$ \\
\hline 205 & 152.70 & 153.19 & 35.99 & 1.50 & 2.01 & 499.87 & 226.82 & 360.00 & 88.56 & 203.255 & SC & $\mathrm{Y}$ & $\mathrm{P}$ \\
\hline 206 & 152.81 & 152.91 & 54.51 & 1.50 & 2.01 & 499.87 & 226.82 & 360.00 & 100.13 & 203.255 & SC & $\mathrm{Y}$ & $\mathrm{P}$ \\
\hline 207 & 152.81 & 152.91 & 19.61 & 1.50 & 2.01 & 499.87 & 226.82 & 360.00 & 93.01 & 203.255 & SC & $\mathrm{Y}$ & $\mathrm{P}$ \\
\hline 208 & 152.50 & 63.70 & 31.09 & 1.50 & 2.01 & 499.87 & 226.82 & 360.00 & 88.11 & 203.255 & SC & $\mathrm{Y}$ & $\mathrm{P}$ \\
\hline 209 & 152.50 & 63.70 & 10.01 & 1.50 & 2.01 & 499.87 & 226.82 & 360.00 & 70.31 & 203.255 & $\mathrm{C}$ & $\mathrm{Y}$ & $\mathrm{P}$ \\
\hline 210 & 152.50 & 63.70 & 16.61 & 1.50 & 2.01 & 499.87 & 226.82 & 360.00 & 75.65 & 203.255 & $\mathrm{C}$ & $\mathrm{Y}$ & $\mathrm{P}$ \\
\hline 211 & 151.00 & 30.78 & 10.01 & 1.50 & 2.01 & 299.97 & 226.82 & 360.00 & 49.84 & 203.255 & $\mathrm{C}$ & $\mathrm{Y}$ & $\mathrm{P}$ \\
\hline 212 & 151.00 & 30.78 & 4.29 & 1.50 & 2.01 & 299.97 & 226.82 & 360.00 & 43.52 & 203.255 & C & $\mathrm{Y}$ & $\mathrm{P}$ \\
\hline 213 & 148.70 & 48.85 & 19.39 & 0.97 & 1.96 & 449.10 & 288.70 & 360.00 & 43.99 & 202.500 & $\mathrm{C}$ & $\mathrm{Y}$ & $\mathrm{P}$ \\
\hline 214 & 148.70 & 73.71 & 19.78 & 0.97 & 2.26 & 449.50 & 288.70 & 360.00 & 41.88 & 202.500 & SC & $\mathrm{Y}$ & $\mathrm{P}$ \\
\hline 215 & 102.70 & 53.00 & 13.20 & 3.00 & 3.50 & 300.00 & 417.00 & 516.75 & 230.00 & 203.255 & SC & $\mathrm{Y}$ & $\mathrm{R}$ \\
\hline 216 & 102.70 & 53.00 & 13.20 & 3.00 & 3.50 & 300.00 & 417.00 & 516.75 & 229.00 & 203.255 & SC & $\mathrm{Y}$ & $\mathrm{R}$ \\
\hline 217 & 102.70 & 53.00 & 13.20 & 3.00 & 3.50 & 300.00 & 417.00 & 516.75 & 230.00 & 203.255 & SC & $\mathrm{Y}$ & $\mathrm{R}$ \\
\hline 218 & 102.70 & 53.00 & 13.20 & 3.00 & 3.50 & 300.00 & 417.00 & 516.75 & 243.00 & 203.255 & SC & $\mathrm{Y}$ & $\mathrm{R}$ \\
\hline 219 & 102.70 & 53.00 & 13.20 & 3.00 & 3.50 & 300.00 & 417.00 & 516.75 & 241.00 & 203.255 & SC & $\mathrm{Y}$ & $\mathrm{R}$ \\
\hline 220 & 102.70 & 53.00 & 13.20 & 3.00 & 3.50 & 300.00 & 417.00 & 516.75 & 236.00 & 203.255 & SC & $\mathrm{Y}$ & $\mathrm{R}$ \\
\hline 221 & 102.70 & 53.00 & 13.20 & 3.00 & 3.50 & 300.00 & 417.00 & 516.75 & 207.00 & 203.255 & SC & $\mathrm{Y}$ & $\mathrm{R}$ \\
\hline 222 & 102.70 & 53.00 & 13.20 & 3.00 & 3.50 & 300.00 & 417.00 & 516.75 & 233.00 & 203.255 & SC & $\mathrm{Y}$ & $\mathrm{R}$ \\
\hline 223 & 102.70 & 53.00 & 13.20 & 3.00 & 3.50 & 300.00 & 417.00 & 516.75 & 229.00 & 203.255 & SC & $\mathrm{Y}$ & $\mathrm{R}$ \\
\hline 224 & 102.70 & 53.00 & 13.20 & 3.00 & 3.50 & 300.00 & 417.00 & 516.75 & 238.00 & 203.255 & SC & $\mathrm{Y}$ & $\mathrm{R}$ \\
\hline 225 & 98.40 & 52.00 & 16.00 & 1.20 & 2.80 & 360.00 & 192.90 & 344.50 & 40.70 & 203.255 & SC & $\mathrm{Y}$ & $\mathrm{P}$ \\
\hline 226 & 98.40 & 52.00 & 16.00 & 1.20 & 2.80 & 360.00 & 192.90 & 344.50 & 42.60 & 203.255 & SC & $\mathrm{Y}$ & $\mathrm{P}$ \\
\hline 227 & 98.40 & 52.00 & 16.00 & 1.20 & 2.80 & 360.00 & 192.90 & 344.50 & 43.20 & 203.255 & SC & $\mathrm{Y}$ & $\mathrm{P}$ \\
\hline 228 & 98.40 & 52.00 & 16.00 & 1.20 & 2.80 & 360.00 & 192.90 & 344.50 & 40.80 & 203.255 & SC & $\mathrm{Y}$ & $\mathrm{P}$ \\
\hline 229 & 98.40 & 52.00 & 16.00 & 1.20 & 2.80 & 360.00 & 192.90 & 344.50 & 39.10 & 203.255 & SC & $\mathrm{Y}$ & $\mathrm{P}$ \\
\hline 230 & 98.40 & 52.00 & 16.00 & 1.20 & 2.80 & 360.00 & 192.90 & 344.50 & 41.90 & 203.255 & SC & $\mathrm{Y}$ & $\mathrm{P}$ \\
\hline 231 & 98.40 & 52.00 & 16.00 & 1.20 & 2.80 & 360.00 & 192.90 & 344.50 & 42.70 & 203.255 & SC & $\mathrm{Y}$ & $\mathrm{P}$ \\
\hline 232 & 98.40 & 52.00 & 16.00 & 1.20 & 2.80 & 360.00 & 192.90 & 344.50 & 42.50 & 203.255 & SC & $\mathrm{Y}$ & $\mathrm{P}$ \\
\hline
\end{tabular}


TABLE 4: Continued.

\begin{tabular}{|c|c|c|c|c|c|c|c|c|c|c|c|c|c|}
\hline $\begin{array}{l}\text { S. } \\
\text { No. }\end{array}$ & $\begin{array}{c}W \\
(\mathrm{~mm})\end{array}$ & $\begin{array}{c}F \\
(\mathrm{~mm})\end{array}$ & $\begin{array}{c}L \\
(\mathrm{~mm})\end{array}$ & $\begin{array}{c}T \\
(\mathrm{~mm})\end{array}$ & $\begin{array}{c}R \\
(\mathrm{~mm})\end{array}$ & $\begin{array}{c}\text { Length } \\
(\mathrm{mm})\end{array}$ & $\begin{array}{c}F_{\mathrm{y}}(\mathrm{N} / \\
\left.\mathrm{mm}^{2}\right)\end{array}$ & $\begin{array}{c}F_{\mathrm{u}}(\mathrm{N} / \\
\left.\mathrm{mm}^{2}\right)\end{array}$ & $\begin{array}{l}P_{\text {Test }} \\
(\mathrm{kN})\end{array}$ & $\begin{array}{l}E(\mathrm{~N} / \\
\left.\mathrm{mm}^{2}\right)\end{array}$ & $\begin{array}{l}\text { Type of } \\
\text { comp. } \\
\text { member }\end{array}$ & $\begin{array}{l}\text { Torsion } \\
\text { allowed }\end{array}$ & $\begin{array}{c}\text { Forming } \\
\text { method }\end{array}$ \\
\hline 233 & 97.60 & 52.00 & 16.00 & 0.80 & 2.00 & 360.00 & 171.30 & 310.05 & 19.70 & 203.255 & SC & $\mathrm{Y}$ & $\mathrm{P}$ \\
\hline 234 & 97.60 & 52.00 & 16.00 & 0.80 & 2.00 & 360.00 & 171.30 & 310.05 & 21.00 & 203.255 & SC & $\mathrm{Y}$ & $\mathrm{P}$ \\
\hline 235 & 97.60 & 52.00 & 16.00 & 0.80 & 2.00 & 360.00 & 171.30 & 310.05 & 20.30 & 203.255 & SC & $\mathrm{Y}$ & $\mathrm{P}$ \\
\hline 236 & 97.60 & 52.00 & 16.00 & 0.80 & 2.00 & 360.00 & 171.30 & 310.05 & 20.70 & 203.255 & SC & $\mathrm{Y}$ & $\mathrm{P}$ \\
\hline 237 & 97.60 & 52.00 & 16.00 & 0.80 & 2.00 & 360.00 & 171.30 & 310.05 & 19.60 & 203.255 & SC & $\mathrm{Y}$ & $\mathrm{P}$ \\
\hline 238 & 97.60 & 52.00 & 16.00 & 0.80 & 2.00 & 360.00 & 171.30 & 310.05 & 20.90 & 203.255 & SC & $\mathrm{Y}$ & $\mathrm{P}$ \\
\hline 239 & 97.60 & 52.00 & 16.00 & 0.80 & 2.00 & 360.00 & 171.30 & 310.05 & 21.10 & 203.255 & SC & $\mathrm{Y}$ & $\mathrm{P}$ \\
\hline 240 & 97.60 & 52.00 & 16.00 & 0.80 & 2.00 & 360.00 & 171.30 & 310.05 & 20.30 & 203.255 & SC & $\mathrm{Y}$ & $\mathrm{P}$ \\
\hline 241 & 102.70 & 53.00 & 13.20 & 3.00 & 3.50 & 300.00 & 417.00 & 516.75 & 239.00 & 203.255 & SC & $\mathrm{Y}$ & $\mathrm{R}$ \\
\hline 242 & 102.70 & 53.00 & 13.20 & 3.00 & 3.50 & 300.00 & 417.00 & 516.75 & 236.00 & 203.255 & SC & $\mathrm{Y}$ & $\mathrm{R}$ \\
\hline 243 & 102.70 & 53.00 & 13.20 & 3.00 & 3.50 & 300.00 & 417.00 & 516.75 & 236.00 & 203.255 & SC & $\mathrm{Y}$ & $\mathrm{R}$ \\
\hline 244 & 102.70 & 53.00 & 13.20 & 3.00 & 3.50 & 300.00 & 417.00 & 516.75 & 234.00 & 203.255 & SC & $\mathrm{Y}$ & $\mathrm{R}$ \\
\hline 245 & 98.40 & 52.00 & 16.00 & 1.20 & 2.80 & 360.00 & 192.90 & 344.50 & 41.70 & 203.255 & SC & $\mathrm{Y}$ & $\mathrm{P}$ \\
\hline 246 & 98.40 & 52.00 & 16.00 & 1.20 & 2.80 & 360.00 & 192.90 & 344.50 & 41.70 & 203.255 & SC & $\mathrm{Y}$ & $\mathrm{P}$ \\
\hline 247 & 98.40 & 52.00 & 16.00 & 1.20 & 2.80 & 360.00 & 192.90 & 344.50 & 41.80 & 203.255 & SC & $\mathrm{Y}$ & $\mathrm{P}$ \\
\hline 248 & 97.60 & 52.00 & 16.00 & 0.80 & 2.00 & 360.00 & 171.30 & 310.05 & 20.40 & 203.255 & SC & $\mathrm{Y}$ & $\mathrm{P}$ \\
\hline 249 & 97.60 & 52.00 & 16.00 & 0.80 & 2.00 & 360.00 & 171.30 & 310.05 & 20.80 & 203.255 & SC & $\mathrm{Y}$ & $\mathrm{P}$ \\
\hline 250 & 97.60 & 52.00 & 16.00 & 0.80 & 2.00 & 360.00 & 171.30 & 310.05 & 20.30 & 203.255 & SC & $\mathrm{Y}$ & $\mathrm{P}$ \\
\hline 251 & 43.76 & 42.49 & 18.71 & 1.85 & 4.15 & 304.80 & 308.33 & 413.40 & 94.25 & 203.255 & SC & $\mathrm{Y}$ & $\mathrm{P}$ \\
\hline 252 & 43.76 & 42.49 & 18.71 & 1.85 & 4.15 & 304.80 & 308.33 & 413.40 & 92.47 & 203.255 & SC & $\mathrm{Y}$ & $\mathrm{P}$ \\
\hline 253 & 43.76 & 42.49 & 18.71 & 1.85 & 4.15 & 304.80 & 308.33 & 413.40 & 101.68 & 203.255 & SC & $\mathrm{Y}$ & $\mathrm{P}$ \\
\hline 254 & 43.76 & 42.49 & 18.71 & 1.85 & 4.15 & 304.80 & 330.10 & 421.39 & 105.47 & 203.255 & SC & $\mathrm{Y}$ & $\mathrm{R}$ \\
\hline 255 & 43.76 & 42.49 & 18.71 & 1.85 & 4.15 & 304.80 & 330.10 & 421.39 & 98.79 & 203.255 & SC & $\mathrm{Y}$ & $\mathrm{R}$ \\
\hline 256 & 44.20 & 42.93 & 18.92 & 2.29 & 3.94 & 177.80 & 304.95 & 429.04 & 133.50 & 203.255 & SC & $\mathrm{Y}$ & $\mathrm{P}$ \\
\hline 257 & 44.20 & 42.93 & 18.92 & 2.29 & 3.94 & 177.80 & 304.95 & 429.04 & 125.94 & 203.255 & SC & $\mathrm{Y}$ & $\mathrm{P}$ \\
\hline 258 & 44.20 & 42.93 & 18.92 & 2.29 & 3.94 & 304.80 & 304.95 & 429.04 & 127.27 & 203.255 & SC & $\mathrm{Y}$ & $\mathrm{P}$ \\
\hline 259 & 44.20 & 42.93 & 18.92 & 2.29 & 3.94 & 304.80 & 304.95 & 429.04 & 123.27 & 203.255 & SC & $\mathrm{Y}$ & $\mathrm{P}$ \\
\hline 260 & 44.20 & 42.93 & 18.92 & 2.29 & 3.94 & 304.80 & 305.02 & 434.35 & 137.06 & 203.255 & SC & $\mathrm{Y}$ & $\mathrm{R}$ \\
\hline 261 & 44.20 & 42.93 & 18.92 & 2.29 & 3.94 & 304.80 & 305.02 & 434.35 & 125.94 & 203.255 & SC & $\mathrm{Y}$ & $\mathrm{R}$ \\
\hline 262 & 300.00 & 75.00 & 20.00 & 2.00 & 3.00 & 500.00 & 242.79 & 344.88 & 119.31 & 200.766 & SC & $\mathrm{Y}$ & $\mathrm{R}$ \\
\hline 263 & 300.00 & 75.00 & 20.00 & 2.00 & 3.00 & 750.00 & 242.79 & 344.88 & 114.08 & 200.766 & $\mathrm{C}$ & $\mathrm{Y}$ & $\mathrm{R}$ \\
\hline 264 & 300.00 & 75.00 & 20.00 & 2.00 & 3.00 & 1000.00 & 242.79 & 344.88 & 109.86 & 200.766 & $\mathrm{C}$ & $\mathrm{Y}$ & $\mathrm{R}$ \\
\hline 265 & 300.00 & 75.00 & 20.00 & 2.00 & 3.00 & 1250.00 & 242.79 & 344.88 & 114.96 & 200.766 & $\mathrm{C}$ & $\mathrm{Y}$ & $\mathrm{R}$ \\
\hline 266 & 300.00 & 75.00 & 20.00 & 2.50 & 3.75 & 500.00 & 282.26 & 372.20 & 159.79 & 203.058 & SC & $\mathrm{Y}$ & $\mathrm{R}$ \\
\hline 267 & 300.00 & 75.00 & 20.00 & 2.50 & 3.75 & 750.00 & 282.26 & 372.20 & 166.97 & 203.058 & $\mathrm{C}$ & $\mathrm{Y}$ & $\mathrm{R}$ \\
\hline 268 & 300.00 & 75.00 & 20.00 & 2.50 & 3.75 & 1000.00 & 282.26 & 372.20 & 182.36 & 203.058 & $\mathrm{C}$ & $\mathrm{Y}$ & $\mathrm{R}$ \\
\hline 269 & 300.00 & 75.00 & 20.00 & 2.50 & 3.75 & 1250.00 & 282.26 & 372.20 & 164.73 & 203.058 & $\mathrm{C}$ & $\mathrm{Y}$ & $\mathrm{R}$ \\
\hline 270 & 300.00 & 75.00 & 20.00 & 3.00 & 4.50 & 500.00 & 258.94 & 350.42 & 156.14 & 206.308 & SC & $\mathrm{Y}$ & $\mathrm{R}$ \\
\hline 271 & 300.00 & 75.00 & 20.00 & 3.00 & 4.50 & 750.00 & 258.94 & 350.42 & 161.37 & 206.308 & $\mathrm{C}$ & $\mathrm{Y}$ & $\mathrm{R}$ \\
\hline 272 & 300.00 & 75.00 & 20.00 & 3.00 & 4.50 & 1000.00 & 258.94 & 350.42 & 178.18 & 206.308 & $\mathrm{C}$ & $\mathrm{Y}$ & $\mathrm{R}$ \\
\hline 273 & 300.00 & 75.00 & 20.00 & 3.00 & 4.50 & 1250.00 & 258.94 & 350.42 & 146.37 & 206.308 & C & $\mathrm{Y}$ & $\mathrm{R}$ \\
\hline
\end{tabular}

Dimensions of lipped channel sections. $W$-web depth in $\mathrm{mm}$. $F$-flange width in $\mathrm{mm}$. $L$-Lip depth in $\mathrm{mm}$. $T$-thickness in mm. $R$-corner radius (inner) in $\mathrm{mm}$. Lipped channel section characteristics. Forming methods. $P$-press brake methods. $R$-cold roll forming method. $P_{\text {Test }}-$ test capacity of section in kN. Material properties. $F_{\mathrm{y}}$-yield strength of steel in $\mathrm{N} / \mathrm{mm}^{2} . F_{\mathrm{u}}$-ultimate strength of steel in $\mathrm{N} / \mathrm{mm}^{2}$. E-modulus of elasticity in $\mathrm{N} / \mathrm{mm}{ }^{2}$. Compression member characteristics. Type of comp. member. C-column, SC-Stub column. Torsion allowed, $\mathrm{Y}-\mathrm{yes}, \mathrm{N}-\mathrm{no}$. Length in mm-centre to centre length for pinned end columns/stub columns.

The algorithm for estimation of $\mathrm{ME}$ and determination of its statistical characteristic is given as follows:

(1) Prepare the test database for ME estimate with exclusion criteria

(2) Filter the test database members (columns and stub columns) for geometric proportions criteria and maximum slenderness ratio limit

(3) Calculate capacities for different governing failure modes for various standards by not considering the safety or partial safety factors present in the model function

(4) Calculate ME as ratio of test to calculated capacity

(5) Plot histograms for $\mathrm{ME}$ and calculate statistical properties

(6) Perform Chi-square goodness-of-fit test for selection of statistical distribution for ME

The probabilistic analysis is carried out for simulation of compression capacity of a typical CFS lipped channel 


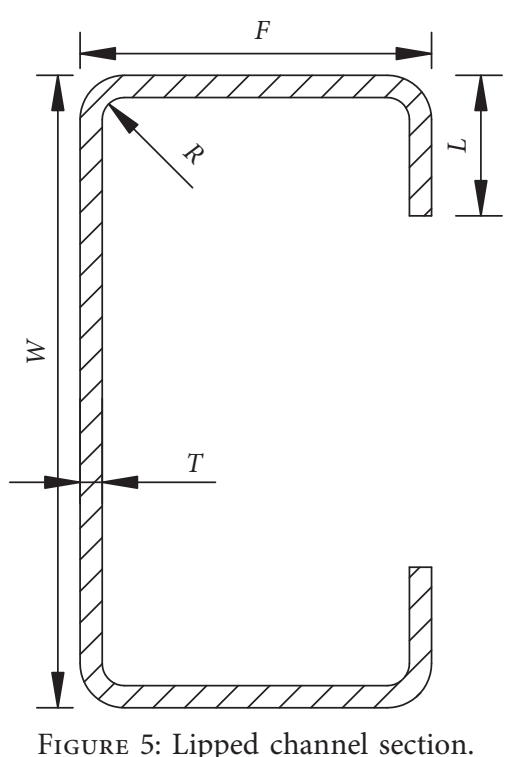

column from the database considering $\mathrm{ME}$ as a random variable. The details of probabilistic analysis and its statistical characteristics are provided in the next section.

Considering the above ME statistics for different buckling failure modes of CFS lipped channel columns, probabilistic analysis is carried out on compression capacity as discussed in the next section.

\section{Probabilistic Analysis of Compression Capacity}

The aim of this section is to bring out the importance of consideration of ME in assuming the distribution of compression capacity. In order to study the effect of ME on compression capacity, simulation of compression capacity is carried out with and without considering $\mathrm{ME}$ as the random variable. The yield strength, $F_{\mathrm{y}}$, ultimate strength, $F_{\mathrm{u}}$, and the modulus of elasticity, $E$, are the material properties of CFS and used in compression capacity estimation of CFS columns as random variables. Ravindra and Galambos [59], and Hess et al. [60], suggested the lognormal distribution for $F_{\mathrm{y}}$ and $E$ and Normal distribution for $F_{\mathrm{u}}$ for high-tensile steel plates. The Coefficient of variation $(\mathrm{COV})$, as per the above studies, for $F_{\mathrm{y}}$ is $0.089, F_{\mathrm{u}}$ is 0.091 , and $E$ is 0.038 , and the same values are adopted in the present study. Simulation of $10^{6}$ cycles are used in present investigations to estimate the statistical properties of compression capacity of a typical CFS lipped channel column (database column no. 18) as per ASCE 10-15 [8], AISI S100-16 [9], AS/NZS 4600: 2018 [10], and EN 1993-1-3:2006 [11] standard, with and without considering $\mathrm{ME}$ as random variable along with the other random variables. The column is selected such that it is having mean ME more than 1.15 and COV more than 0.25. The column is critical in flexure torsion mode of buckling as per the abovementioned standards. The histograms along with statistical properties of simulated compression capacity for abovementioned standards estimated with and without $\mathrm{ME}$ as random variable are presented in Figures 9-11.
Further, to predict statistical distributions, Chi-square goodness-of-fit test has been performed on simulated compression capacity, and the results of this test are given in Table 8.

The abovementioned results of modelling error estimation and probabilistic analysis are discussed in the following section.

\section{Results and Discussions}

The ME is estimated for various failure modes considered in ASCE 10-15, AISI S100-16, AS/NZS 4600: 2018, and EN 1993-1-3: 2006 standards for the database columns. The statistical details of $\mathrm{ME}$ and results of Chi-square goodnessof-fit test are presented in Figures 6-8 and Tables 6 and 7, respectively. The distributions of simulated compression capacity based on probabilistic analysis and corresponding results of Chi-square goodness-of-fit tests are indicated in Figures 9-11 and Table 8, respectively. Based on these results, the following observations are made.

\subsection{Modelling Error}

7.1.1. Mean. The mean ME for column buckling in flexure is close to 0.9 for ASCE 10-15 and AISI S100-16 \& AS/NZS 4600:2018 standard, whereas the same for EN 1993-1-3:2006 standard is 0.95 . In case of flexural torsional buckling, the mean ME is close to 1.2, irrespective of the design standard. For local buckling, the mean ME is 0.92 for ASCE 10-15 standard and 0.82 for AISI S100-16 \& AS/NZS 4600:2018 standard. The ASCE 10-15 standard is having stringent criteria for dimensional proportions with remote chances of distortional buckling and hence does not provide any guideline for it. As per AISI S100-16 \& AS/NZS 4600:2018 standard, the mean ME in distortional buckling is 1.34, which is a high value. For EN 1993-1-3:2006 standard, the combined model function is provided for the local and distortional buckling and it gives mean ME as 1.11.

In case of stub columns, the mean ME for yield failure of cross section is 1.11 for ASCE 10-15 and 1.02 for AISI S10016 \& AS/NZS 4600:2018 standard. EN 1993-1-3:2006 standard does not provide separate guidelines for yield failure of the cross section. For local buckling, the mean model error is 0.97 and 0.88 , respectively, for the ASCE 1015 and AISI S100-16 \& AS/NZS 4600:2018 standard. The mean ME in distortional buckling for AISI S100-16 \& AS/ NZS 4600:2018 standard is 0.95. The combined mode of failure for local and distortional buckling is suggested in EN 1993-1-3:2006 standard, and the mean ME in this mode is 1.02 .

It is observed that for columns, the mean MEs are within $\pm 10 \%$ variation for the single mode failures viz. flexure and local buckling. In case of combined failure mode in flexure and torsion, the variation is around $+20 \%$ and for distortion buckling it is $+34 \%$. This indicates requirement of extensive experimental research in combined and distortion buckling modes. For stub columns, a good estimate of $\pm 10 \%$ of mean ME was observed irrespective of the design standards and failure modes; except for the local buckling as per AISI 
TABle 5: Salient features of design standards.

Failure mode

\section{Design standard}

AISI S100-16 and AS/NZS: 46002018
SSRC (1966) equation is used for columns in inelastic and Euler's equation for columns in elastic range of buckling. (i) The elastic critical buckling load for a long column is determined by the Euler's equations.

(ii) For locally stable columns, the AISC LRFD specification (1993)

equation is adopted for elastic and inelastic ranges of buckling.
As per this code the local buckling strength is not equal to torsional

Torsion buckling strength. Hence, torsional-flexural buckling strength is determined.
The torsional buckling in the elastic range is computed based on the equation provided by Winter [53] for elastic critical stress.
EN 1993-1-3: 2006

The resistance of compressed members is based on the "European design buckling curves" (ECCS, 1978) that relate the reduction factor to the nondimensional slenderness. These (five) curves were the result of an extensive experimental and numerical research programme (ECCS, 1976),

conducted on HR and welded sections, that accounted for all imperfections in real compressed members (initial outof-straightness, eccentricity of the applied loads, residual stresses). The analytical formulation of the buckling curves was derived by Maquoi \& Rondal [33], and is based on the Ayrton-Perry formula, considering an initial sinusoidal deformed configuration corresponding to an "equivalent initial deformed configuration" where the amplitude was calibrated in order to reproduce the effect of all imperfections.

For members with "point-symmetric" open cross sections (e.g. Z-purlin with equal flanges), the possibility that the resistance of the member to torsional buckling might be less than its resistance on flexural buckling is considered in this code.

The governing elastic flexural-

The design compressive stress for torsional-flexural buckling strength is determined using an equivalent radius of gyration. torsional buckling load of a column can be found from the equation suggested by Chajes and Winter [54]; Chajes et al. [55]; Yu and LaBoube [56].
For members with mono-symmetric open cross-sections, the possibility of the resistance of the member to torsional-flexural buckling might be less than its resistance to flexural buckling is considered in this code.
If element slenderness ratio is not small enough to develop uniform design stress distributed over full cross section, then the post buckling strength of element which buckles prematurely is taken into account by using an effective width of an element in determining the area of the member cross section. The effective width of an element is the width, which gives the same resultant force of a uniformly distributed design stress, as the nonuniform stress develops in the entire element in the post-buckled
In this code, the effective width Method's approach to local buckling adopted, whereby 'ineffective' portions is adopted. It conceptualizes the member as a collection of "elements" and investigates local buckling of each element separately.

The effective width method determines a plate buckling coefficient, $k$, for each element, then buckling stress, and finally the effective width.
An effective width approach is of a cross section are removed and section properties may be determined based on the remaining effective portions.

In this standard, the local and distortional buckling modes for cross sections with edge stiffeners are considered together while estimating the resistance. 
TABle 5: Continued.

Failure mode

\section{Design standard}

AISI S100-16 and AS/NZS: 46002018
Distortional buckling is an instability that may occur in members with edge-stiffened flanges, such as lipped

$\mathrm{C}$ and $\mathrm{Z}$-sections. This buckling mode is characterized by instability of the entire flange, as the flange along with the edge stiffener rotates about the junction of the compression flange and the web. The expressions in this specification are derived by Schafer [57] and verified for complex stiffeners by $\mathrm{Yu}$ and Schafer [58].
Very short, compact column under an axial load may fail by yielding.

Yielding

Not indicated
Hence, the yield load determined by multiplying the gross area with yield strength.
EN 1993-1-3: 2006

EN1993-1-3 does not provide explicit provisions for distortional buckling. However, a calculation procedure is obtained from the interpretation of the rules given in the code for plane elements with edge or intermediate stiffeners in compression. The design of compression elements with either edge or intermediate stiffeners is based on the assumption that the stiffener behaves as a compression member with continuous partial restraint. This restraint has a spring stiffness that depends on the boundary conditions and the flexural stiffness of the adjacent plane elements of the cross section. The spring stiffness of the stiffener may be determined by applying a unit load per unit length to the cross section at the location of the stiffener. The rotational spring stiffness characterizes the bending stiffness of the web part of the section.

The design resistance is computed by multiplying the gross area with increased basic yield stress for the contribution from difference of average and basic yield stress reduced by a factor based on the ratio of relative slenderness for elements.

(i) For stiffened element in compression, $\mathrm{w} / \mathrm{t} \leq 500$,

(i) For stiffened element in compression, $\mathrm{w} / \mathrm{t} \leq 500$,

(i) For elements supported on both Maximum element the longitudinal edges, $w / t \leq 60$ and slenderness ratio $(\mathrm{w} / \mathrm{t})$ (ii) for elements supported on one longitudinal edge, $\mathrm{w} / \mathrm{t} \leq 25$ $\mathrm{w} / \mathrm{t}=$ flat width to thickness ratio. (ii) for edge stiffened element in compression $\mathrm{w} / \mathrm{t} \leq 90$ for $I_{\mathrm{s}} \geq / I_{\mathrm{a}}$ and

$\mathrm{w} / \mathrm{t} \leq 60$ for $I_{\mathrm{s}}<I_{\mathrm{a}}$, and (iii) for unstiffened element in compression $\mathrm{w} / \mathrm{t} \leq 60$ $\mathrm{w} / \mathrm{t}=$ flat width to thickness ratio. (ii) for edge stiffened element in compression a) for element $w / t \leq 60$ for stiffener $w / t \leq 50$, and (iii) for unstiffened element in compression $\mathrm{w} / \mathrm{t} \leq 50$

$\mathrm{w} / \mathrm{t}=$ out to out width to thickness ratio.

Other Considerations

Material strength

As per ASTM standards with yield strength up to $448 \mathrm{MPa}$
As per ASTM standards with yield strength up to $380 \mathrm{MPa}$
As per EN standards with basic yield strength up to $460 \mathrm{MPa}$ 
TABle 5: Continued.

Failure mode
Design standard

AISI S100-16 and AS/NZS: 46002018
EN 1993-1-3: 2006
Increase in yield strength of material due to cold-working
Not considered.
At Cornell University, the influence of cold work on mechanical properties was investigated by Chajes et al. [2], Karren, [3], and Karren and Winter [4]. It was found that the changes of mechanical properties due to cold-stretching are caused mainly by strain-hardening and strain-aging, Chajes et al. [2]. Cornell research also revealed that the effects of cold work on the mechanical properties of corners usually depend on (1) the type of steel, (2) the type of stress (compression or tension), (3) the direction of stress with respect to the direction of cold work (transverse or longitudinal), (4) the Fu/Fy ratio, (5) the inside radius-to-thickness ratio $(\mathrm{R} / \mathrm{t})$, and (6) the amount of cold work.

Investigating the influence of cold work, Karren derived the equations for the ratio of corner yield stress-tovirgin yield stress [3]. With regard to the full-section properties, the tensile yield stress of the full section approximated by using a weighted average is used in this specification. Good agreements between the computed and the tested stress-strain characteristics for a channel section and a joist chord section were demonstrated by Karren and Winter [4]
S100-16 (2016) \& AS/NZS 4600:2018 (2018) standards, the variation in mean $\mathrm{ME}$ is $12 \%$.

7.1.2. COV. The COV of ME for columns as per ASCE 10-15 and AISI S100-16 \& AS/NZS 4600:2018 standards is close to 0.15 in flexural and local buckling modes, whereas it is 0.26 in flexural torsional buckling mode. The same is 0.28 for distortional buckling mode as per AISI S100-16 \& AS/NZS 4600:2018 standard. In case of EN 1993-1-3:2006 standard, the $\mathrm{COV}$ is 0.27 and 0.29 , respectively, for the flexural and flexural-torsional buckling modes, and in combined local and distortion buckling mode, it is 0.01 .

In case of stub columns, low values of COV are observed for ASCE 10-15 and AISI S100-16 \& AS/NZS 4600:2018 standards, i.e., 0.08 in yielding and 0.05 in local buckling. For distortional buckling, the COV is 0.09 for AISI S100-16 \& AS/NZS 4600:2018 standards and, it is 0.01 for the combined mode of local and distortion buckling as per EN 1993-1-3: 2006 standard.

It indicates that the higher uncertainties are involved in prediction of column buckling capacities in comparison
The increased yield strength due to cold forming may be taken into account if in axially loaded members in which the effective cross-sectional area equals the gross area, and in determining the effective area, the yield strength should be taken as basic yield strength.

7.1.3. Skewness. The nonzero values of skewness observed in all the buckling modes indicates a non-Gaussian distribution for ME data. Further, most of the skewness values are positive indicating positively skewed or right-skewed distribution in which maximum number of values are clustered around the left tail of the distribution while the right tail of the distribution is longer and the same is observed in Figures 6-8. For few failure modes (viz. local buckling of stub columns for ASCE 10-15 standard, yielding of stub columns for AISI S100-16 \& AS/NZS 4600:2018 standard, and local and distortional buckling of columns for EN 1993- 
TABLE 6: Statistical properties of modelling error of test database compression members.

\begin{tabular}{|c|c|c|c|c|c|c|c|c|}
\hline \multirow{3}{*}{$\begin{array}{l}\text { Design } \\
\text { standard }\end{array}$} & \multirow{3}{*}{$\begin{array}{l}\text { Statistical } \\
\text { property }\end{array}$} & \multicolumn{7}{|c|}{ Compression members \& failure modes } \\
\hline & & \multicolumn{4}{|c|}{ Columns } & \multicolumn{3}{|c|}{ Stub columns } \\
\hline & & $\begin{array}{l}\text { Flexural } \\
\text { buckling }\end{array}$ & $\begin{array}{l}\text { Flexure- } \\
\text { torsion } \\
\text { buckling }\end{array}$ & $\begin{array}{c}\text { Local } \\
\text { buckling }\end{array}$ & Distortion buckling & Yielding & $\begin{array}{c}\text { Local } \\
\text { buckling }\end{array}$ & Distortion buckling \\
\hline \multirow{6}{*}{ ASCE $10-15$} & & \multicolumn{7}{|c|}{ Number of specimens from database critical in Failure mode } \\
\hline & $\operatorname{Nos}^{@}$ & 14 & 33 & 10 & \multirow{5}{*}{$\begin{array}{l}\text { Failure mode not } \\
\text { available in this } \\
\text { standard }\end{array}$} & 27 & 10 & \multirow{5}{*}{$\begin{array}{c}\text { Failure mode not } \\
\text { considered in this } \\
\text { standard }\end{array}$} \\
\hline & Mean & 0.899 & 1.154 & 0.915 & & 1.108 & 0.969 & \\
\hline & $\mathrm{COV}$ & 0.150 & 0.257 & 0.132 & & 0.082 & 0.043 & \\
\hline & SK & 2.018 & 1.934 & 0.962 & & 0.476 & -0.388 & \\
\hline & $\mathrm{CU}$ & 8.131 & 5.828 & 2.659 & & 2.238 & 2.566 & \\
\hline \multirow{6}{*}{$\begin{array}{l}\text { AISI S100-16 \& } \\
\text { AS/NZS 4600: } \\
2018\end{array}$} & & \multicolumn{7}{|c|}{ Number of specimens from database critical in Failure mode } \\
\hline & Nos ${ }^{@}$ & 14 & 34 & 23 & 40 & 17 & 32 & 42 \\
\hline & Mean & 0.870 & 1.198 & 0.821 & 1.338 & 1.017 & 0.884 & 0.951 \\
\hline & $\mathrm{COV}$ & 0.149 & 0.262 & 0.164 & 0.279 & 0.081 & 0.049 & 0.091 \\
\hline & SK & 2.331 & 1.864 & 1.036 & 0.155 & -0.228 & 1.669 & 0.134 \\
\hline & $\mathrm{CU}$ & 9.550 & 5.544 & 4.034 & 1.813 & 2.289 & 9.465 & 4.677 \\
\hline \multicolumn{9}{|c|}{ Number of specimens from database critical in Failure mode } \\
\hline \multirow{5}{*}{$\begin{array}{l}\text { EN 1993-1-3: } \\
2006\end{array}$} & Nos ${ }^{@}$ & \multicolumn{7}{|c|}{57 Number or specimens irom database critical in Fallure mode } \\
\hline & Mean & 0.942 & 1.225 & & 1.113 & - & \multicolumn{2}{|r|}{1.024} \\
\hline & $\mathrm{COV}$ & 0.227 & 0.289 & & 0.012 & - & \multicolumn{2}{|r|}{0.120} \\
\hline & SK & 0.810 & 1.229 & & -1.682 & - & \multicolumn{2}{|r|}{0.147} \\
\hline & $\mathrm{CU}$ & 2.325 & 3.661 & & - & - & \multicolumn{2}{|r|}{3.584} \\
\hline
\end{tabular}

${ }^{\circledR}$ Number of Specimens from the test database critical in particular failure mode. EN 1993-1-3:2006 considers combined failure mode as local and distortion buckling.

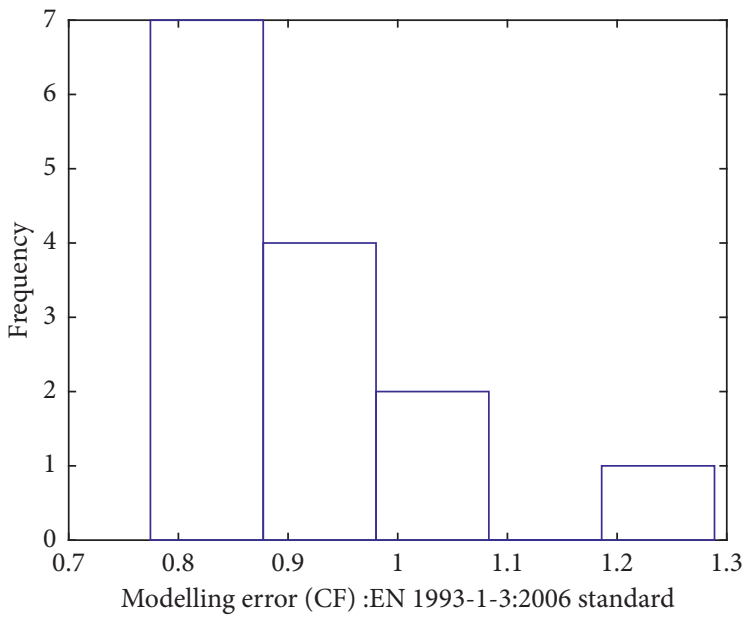

(a)

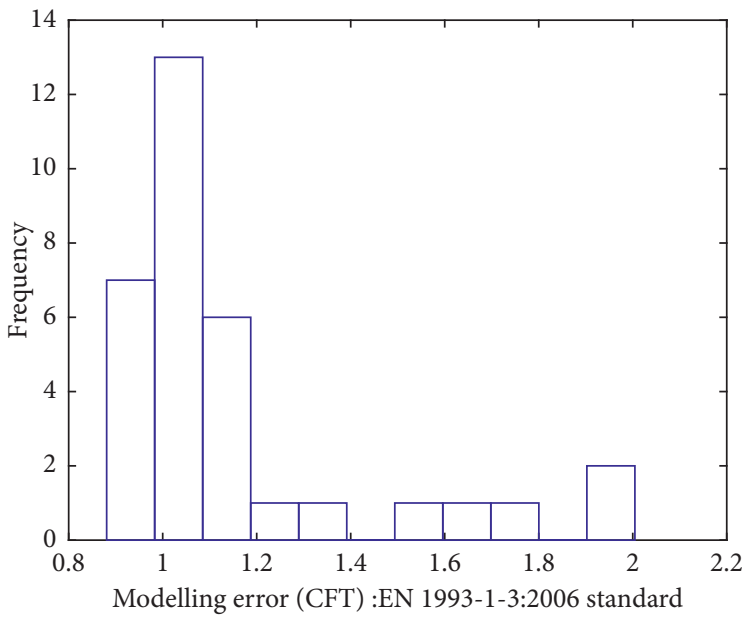

(b)

Figure 6: Continued. 


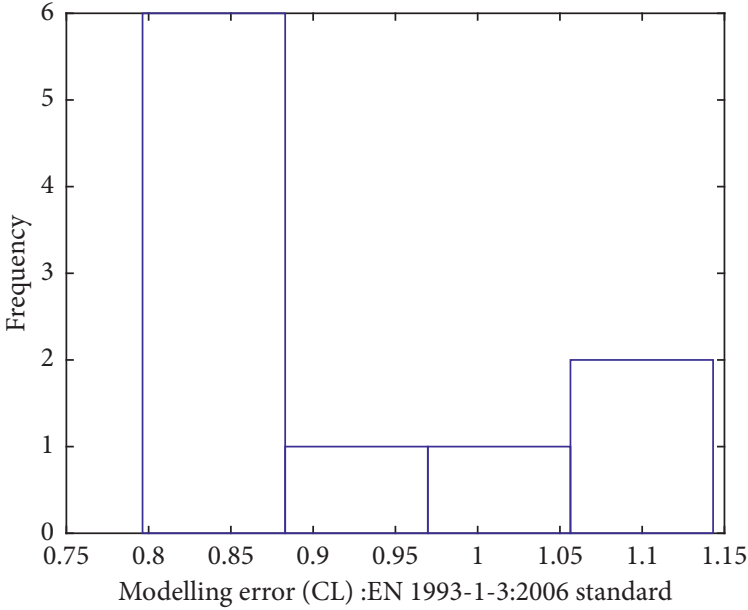

(c)

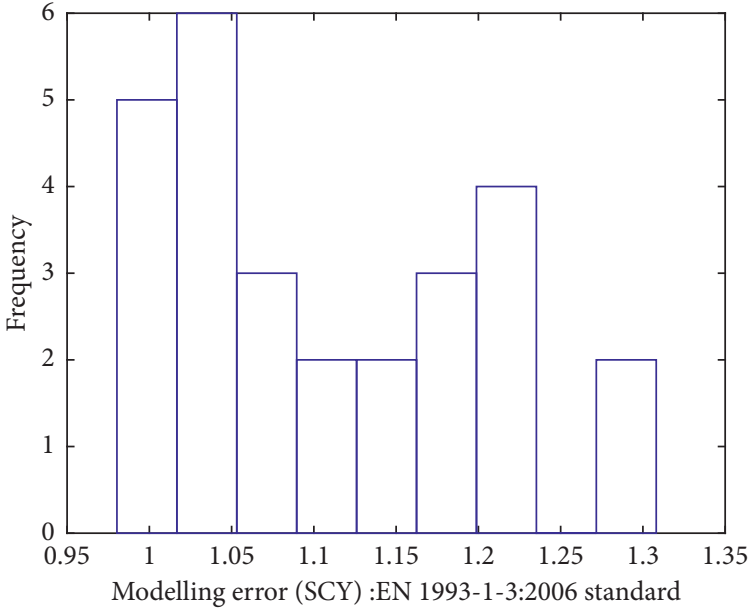

(d)

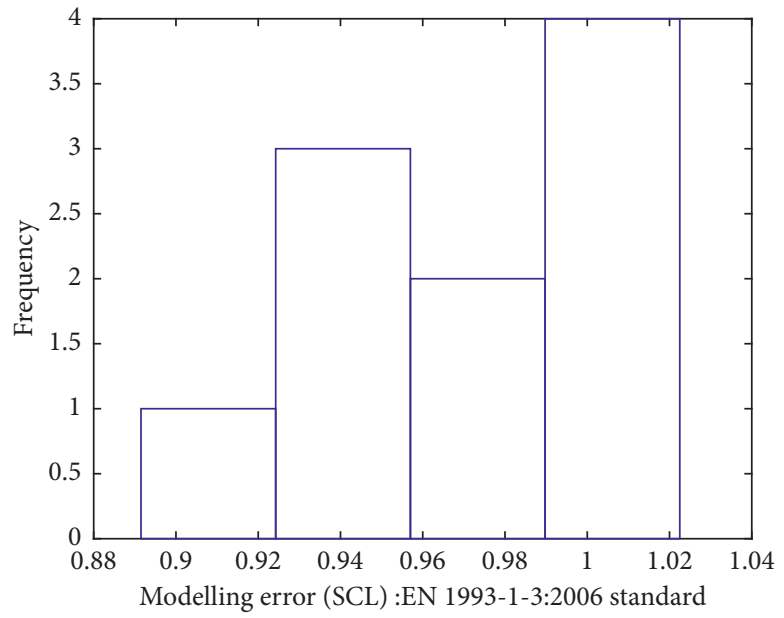

(e)

Figure 6: ME histograms for various failure modes for database member as per ASCE 10-15 Standard. (a) CF-column flexure; (b) CFT—column flexure-torsion; (c) CL_column local buckling; (d) SCY—stub column yielding; (e) SCL—stub column local buckling.

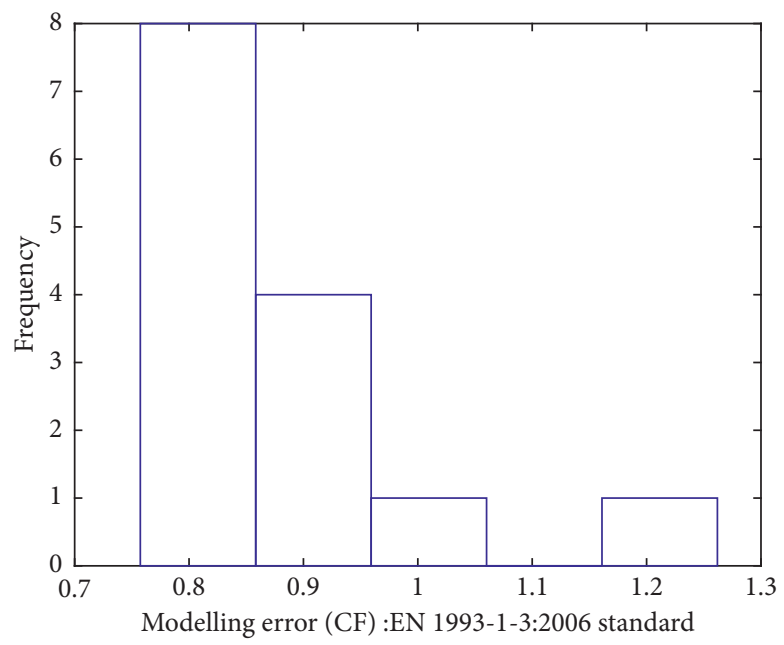

(a)

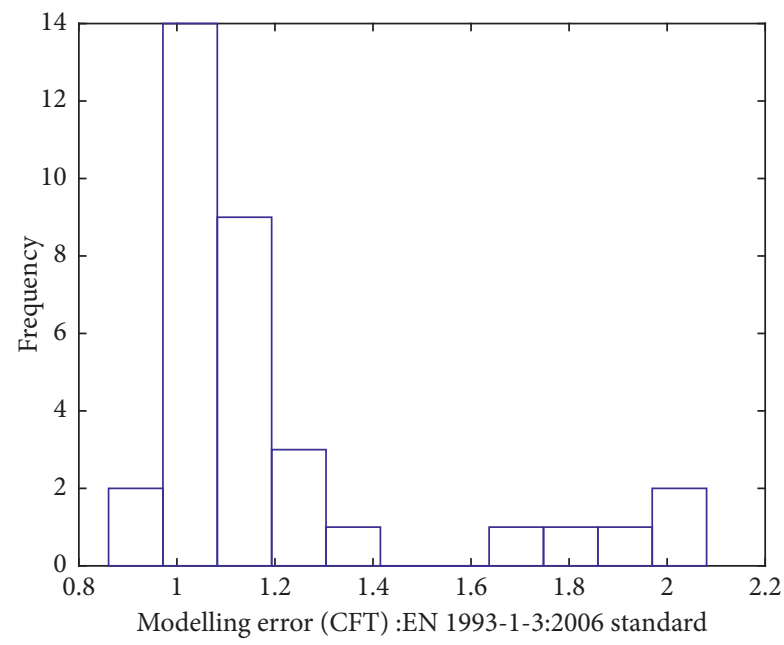

(b)

Figure 7: Continued. 


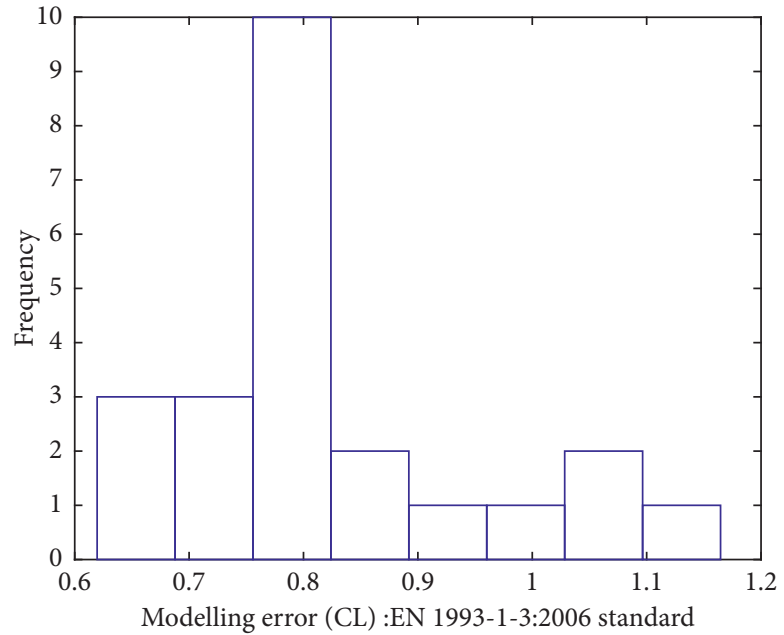

(c)

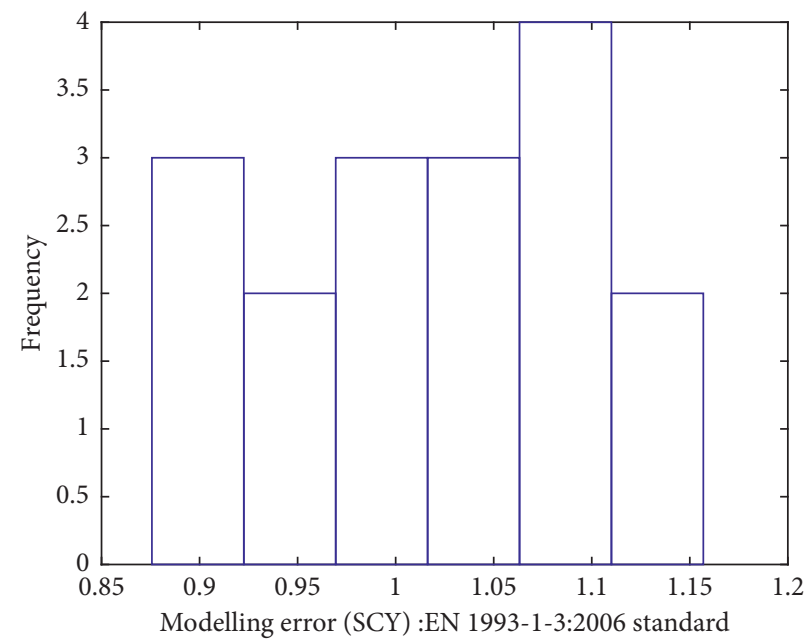

(e)

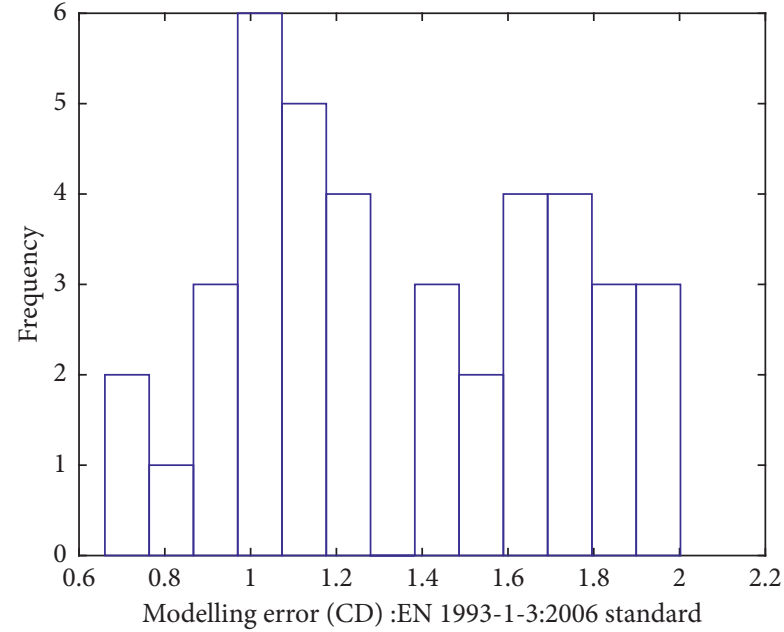

(d)

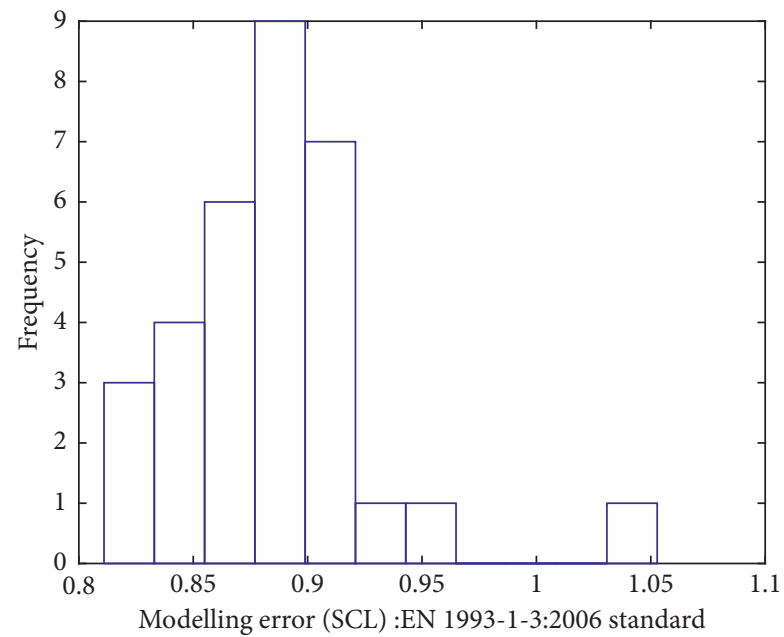

(f)

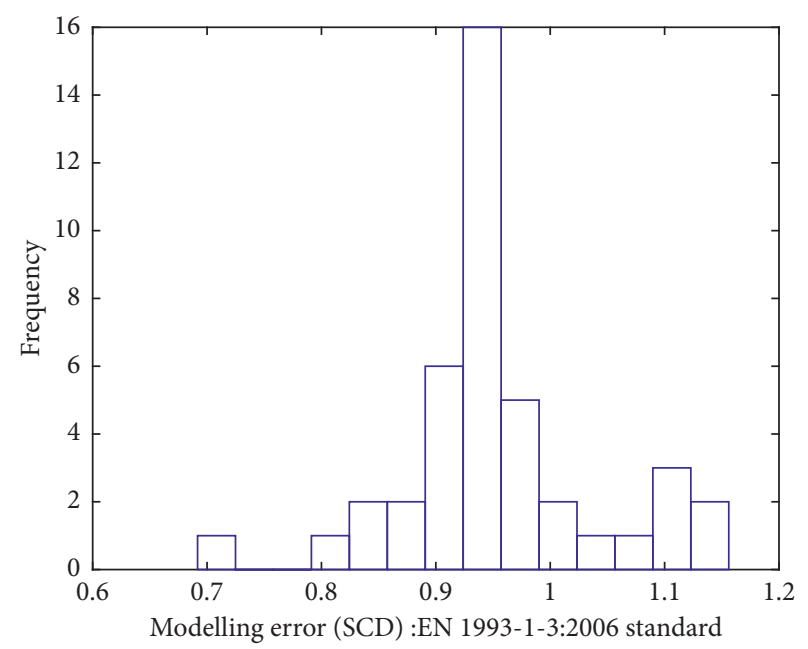

(g)

FIGURE 7: ME histograms for various failure modes for database member as per AISI S100-16 \& AS/NZS 4600: 2018 Standards. (a) $\mathrm{CF}$-column flexure; (b) CFT—column flexure-torsion; (c) CL_column local buckling; (d) CD—column distortion buckling; (e) SCY_stub column yielding; (f) SCL_stub column local buckling; (g) SCD—stub column distortion buckling. 


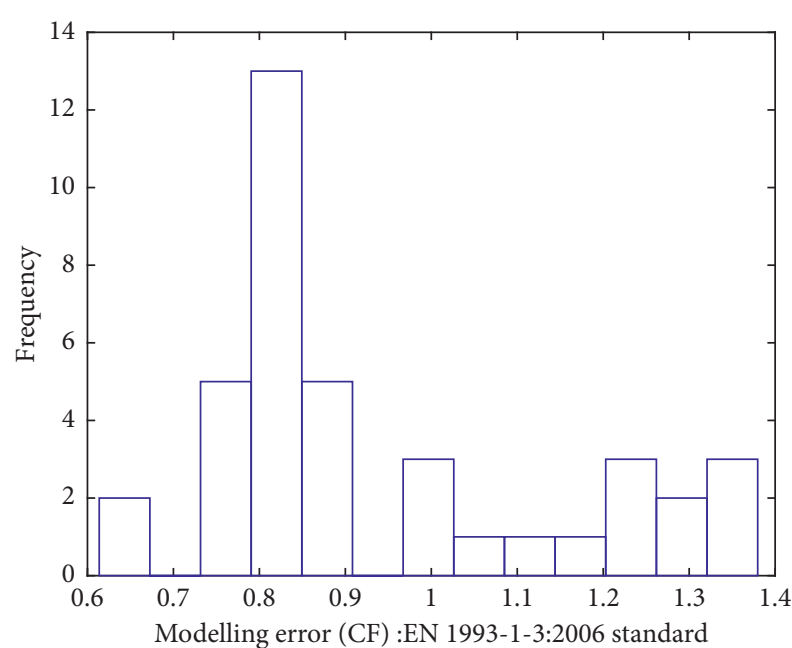

(a)

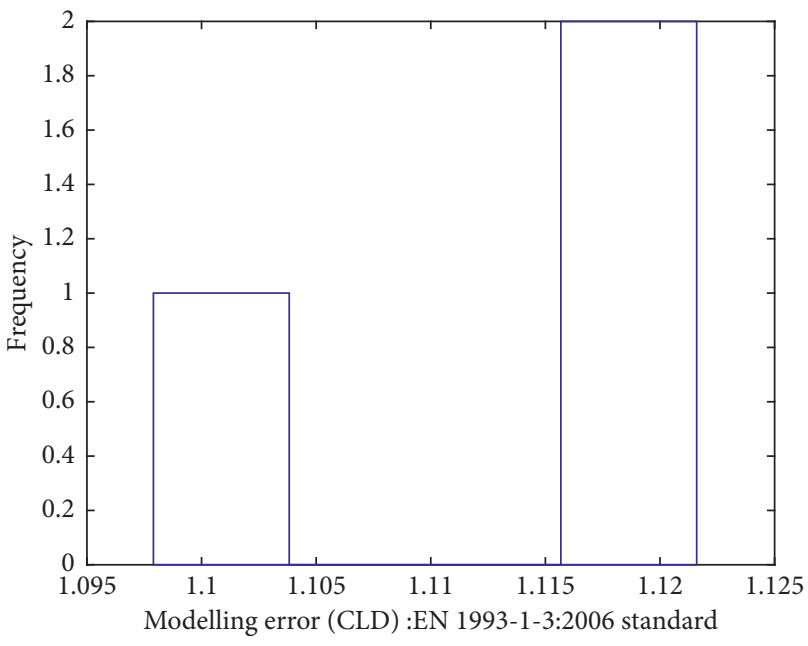

(c)

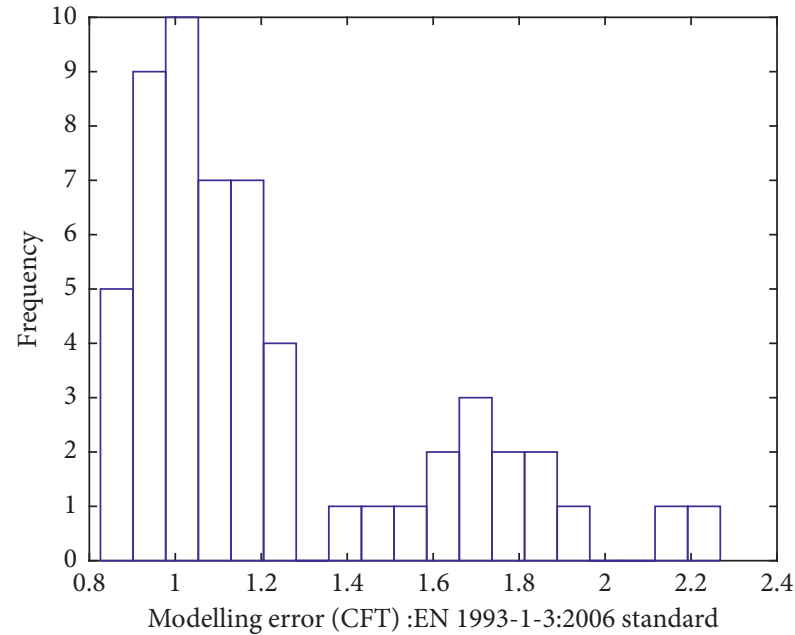

(b)

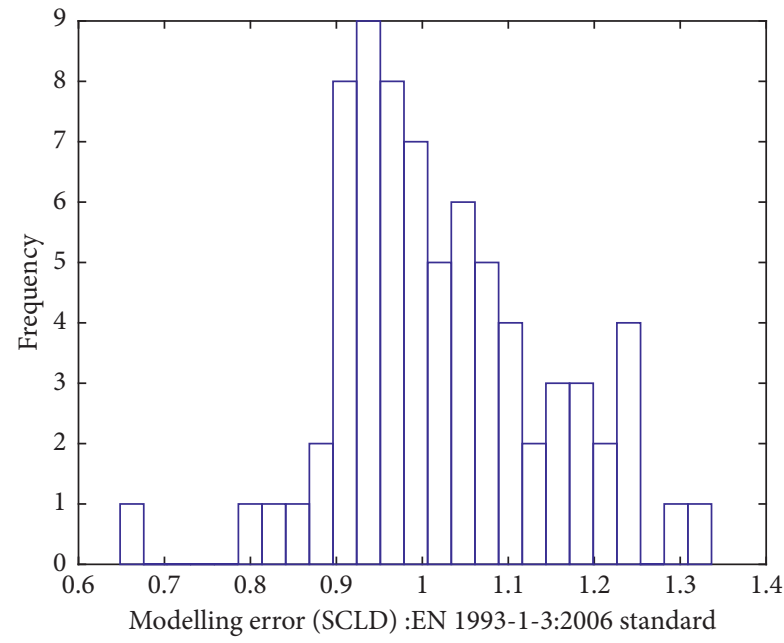

(d)

FIGURE 8: ME histograms for various failure modes for database member as per EN 1993-1-3:2006 Standard. (a) CF—column flexure; (b) CFT—column flexure-torsion; (c) CLD—column local and distortion buckling; (d) SCLD—stub column local and distortional buckling.

TABLE 7: Results of Chi-square goodness-of-fit test for modelling error of test database compression members.

\begin{tabular}{|c|c|c|c|c|c|c|c|}
\hline \multirow{2}{*}{ Design standard } & \multirow{2}{*}{$\begin{array}{c}\text { Type of compression } \\
\text { member }\end{array}$} & \multirow{2}{*}{ Failure mode } & \multirow{2}{*}{ Nos@ } & \multirow{2}{*}{$\begin{array}{c}\text { Assumed } \\
\text { distribution (DOF) }\end{array}$} & \multirow{2}{*}{$\begin{array}{l}\text { Computed Chi- } \\
\text { square value }\end{array}$} & \multicolumn{2}{|c|}{$\begin{array}{l}\text { Allowable Chi- } \\
\text { square value }\end{array}$} \\
\hline & & & & & & $\begin{array}{c}\text { At } \\
\alpha=5 \%\end{array}$ & $\begin{array}{c}\text { At } \\
\alpha=1 \%\end{array}$ \\
\hline \multirow{15}{*}{ ASCE $10-15$} & \multirow{9}{*}{ Column } & \multirow{3}{*}{ Flexural buckling } & \multirow{3}{*}{14} & Normal (2) & 0.556 & \multirow{3}{*}{5.99} & \multirow{3}{*}{9.21} \\
\hline & & & & Lognormal (2) & 0.354 & & \\
\hline & & & & Uniform (2) & 0.722 & & \\
\hline & & \multirow{4}{*}{$\begin{array}{l}\text { Flexure-torsion } \\
\text { buckling }\end{array}$} & \multirow{4}{*}{33} & Normal (8) & 1.837 & \multirow{4}{*}{15.51} & \multirow{3}{*}{20.09} \\
\hline & & & & Lognormal (8) & 1.057 & & \\
\hline & & & & Uniform (8) & 1.523 & & \\
\hline & & & & Normal (1) & 0.875 & & \\
\hline & & \multirow[t]{3}{*}{ Local buckling } & \multirow[t]{3}{*}{10} & Lognormal (1) & 0.753 & \multirow[t]{3}{*}{3.84} & \multirow[t]{3}{*}{6.63} \\
\hline & & & & Uniform (1) & 0.853 & & \\
\hline & \multirow{6}{*}{ Stub column } & & & Normal (6) & 0.572 & & \\
\hline & & Yielding & 27 & Lognormal (6) & 0.525 & \multirow[t]{3}{*}{12.59} & \multirow[t]{3}{*}{16.81} \\
\hline & & \multirow{4}{*}{ Local buckling } & \multirow{4}{*}{10} & Uniform (6) & 0.311 & & \\
\hline & & & & Normal (1) & 0.223 & & \\
\hline & & & & Lognormal (1) & 0.242 & \multirow[t]{2}{*}{3.84} & \multirow[t]{2}{*}{6.63} \\
\hline & & & & Uniform (1) & 0.226 & & \\
\hline
\end{tabular}


TABle 7: Continued.

\begin{tabular}{|c|c|c|c|c|c|c|c|}
\hline \multirow{2}{*}{ Design standard } & \multirow{2}{*}{$\begin{array}{c}\text { Type of compression } \\
\text { member }\end{array}$} & \multirow{2}{*}{ Failure mode } & \multirow{2}{*}{ Nos@ } & \multirow{2}{*}{$\begin{array}{c}\text { Assumed } \\
\text { distribution (DOF) }\end{array}$} & \multirow{2}{*}{$\begin{array}{l}\text { Computed Chi- } \\
\text { square value }\end{array}$} & \multicolumn{2}{|c|}{$\begin{array}{l}\text { Allowable Chi- } \\
\text { square value }\end{array}$} \\
\hline & & & & & & $\begin{array}{c}\text { At } \\
\alpha=5 \%\end{array}$ & $\begin{array}{c}\text { At } \\
\alpha=1 \%\end{array}$ \\
\hline \multirow{21}{*}{$\begin{array}{l}\text { AISI S100-16 \& AS/ } \\
\text { NZS 4600: } 2018\end{array}$} & \multirow{12}{*}{ Column } & \multirow{3}{*}{ Flexural buckling } & \multirow{3}{*}{14} & Normal (2) & 0.820 & \multirow{3}{*}{5.99} & \multirow{3}{*}{9.21} \\
\hline & & & & Lognormal (2) & 0.523 & & \\
\hline & & & & Uniform (2) & 0.986 & & \\
\hline & & \multirow{4}{*}{$\begin{array}{l}\text { Flexure-torsion } \\
\text { buckling }\end{array}$} & \multirow{4}{*}{34} & Normal (8) & 1.801 & \multirow{3}{*}{15.51} & \multirow{3}{*}{20.09} \\
\hline & & & & Lognormal (8) & 1.116 & & \\
\hline & & & & Uniform (8) & 1.647 & & \\
\hline & & & & Normal (5) & 0.576 & & \\
\hline & & \multirow[t]{3}{*}{ Local buckling } & \multirow[t]{3}{*}{23} & Lognormal (5) & 0.477 & \multirow[t]{3}{*}{11.07} & \multirow{2}{*}{15.09} \\
\hline & & & & Uniform (5) & 0.839 & & \\
\hline & & & & Normal (10) & 0.379 & & \multirow{4}{*}{23.21} \\
\hline & & \multirow[t]{3}{*}{ Distortion buckling } & \multirow[t]{3}{*}{40} & Lognormal (10) & 0.442 & \multirow{3}{*}{18.31} & \\
\hline & & & & Uniform (10) & 0.244 & & \\
\hline & \multirow{9}{*}{ Stub column } & & & Normal (3) & 0.190 & & \\
\hline & & Yielding & 17 & Lognormal (3) & 0.204 & \multirow[t]{3}{*}{7.81} & \multirow[t]{3}{*}{11.34} \\
\hline & & & & Uniform (3) & 0.060 & & \\
\hline & & & & Normal (8) & 3.455 & & \\
\hline & & Local buckling & 32 & Lognormal (8) & 1.682 & 15.51 & 20.09 \\
\hline & & & & Uniform (8) & 0.902 & & \\
\hline & & & & Normal (11) & 0.811 & & \\
\hline & & Distortion buckling & 42 & Lognormal (11) & 1.031 & 19.68 & 24.73 \\
\hline & & & & Uniform (11) & 1.322 & & \\
\hline & & & & Normal (10) & 1.183 & & \\
\hline & & Flexural buckling & 39 & Lognormal (10) & 0.978 & 18.31 & 23.21 \\
\hline & & & & Uniform (10) & 1.156 & & \\
\hline & & & & Normal (16) & 0.856 & & \\
\hline & Column & Flexure-torsion & 57 & Lognormal (16) & 0.473 & 26.30 & 32.00 \\
\hline & & & & Uniform (16) & 0.902 & & \\
\hline EN 1993-1-3: 2006 & & & & Normal (0) & 1.260 & & \\
\hline & & Local and distortion & 3 & Lognormal (0) & 1.266 & - & - \\
\hline & & buckling & & Uniform (0) & 1.717 & & \\
\hline & & & & Normal (22) & 0.408 & & \\
\hline & Stub column & Local and distortion & 74 & Lognormal (22) & 1.023 & 33.92 & 40.29 \\
\hline & & & & Uniform (22) & 0.781 & & \\
\hline
\end{tabular}

${ }^{\circledR}$ Number of Specimens from the test database critical in particular failure mode.

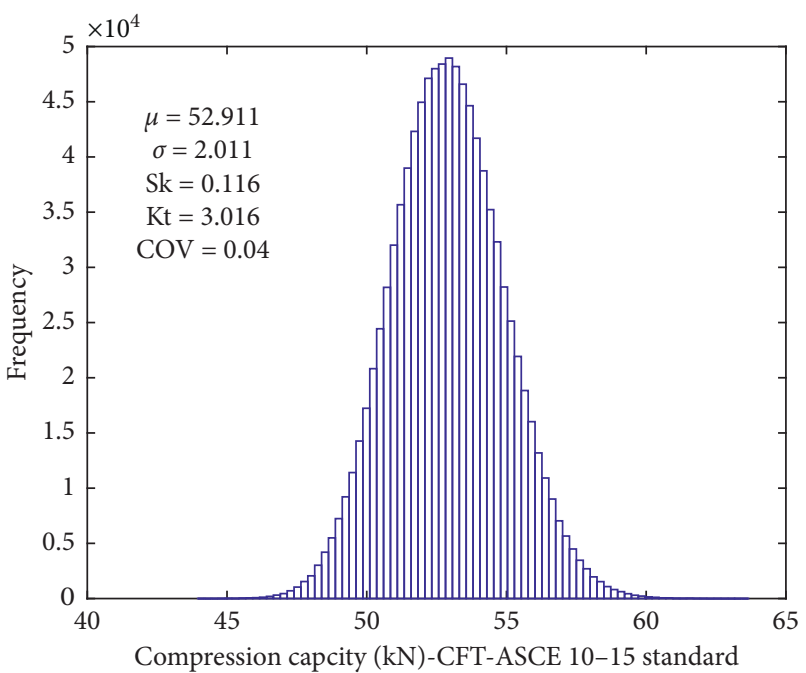

(a)

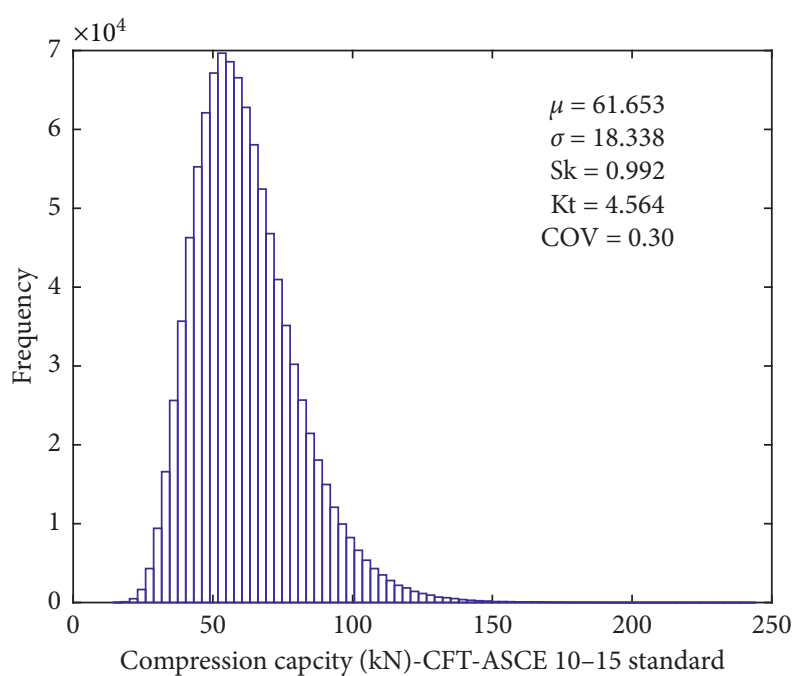

(b)

FIGURE 9: Compression capacity histograms for ASCE 10-15 Standard: (a) compression capacity without ME; (b) compression capacity with ME. CFT_Column flexure-torsion buckling mode, $\mu$-Mean, $\sigma$-Std. deviation, Sk-Skewness, Kt-Kurtosis, and COV-Coefficient of variation. 


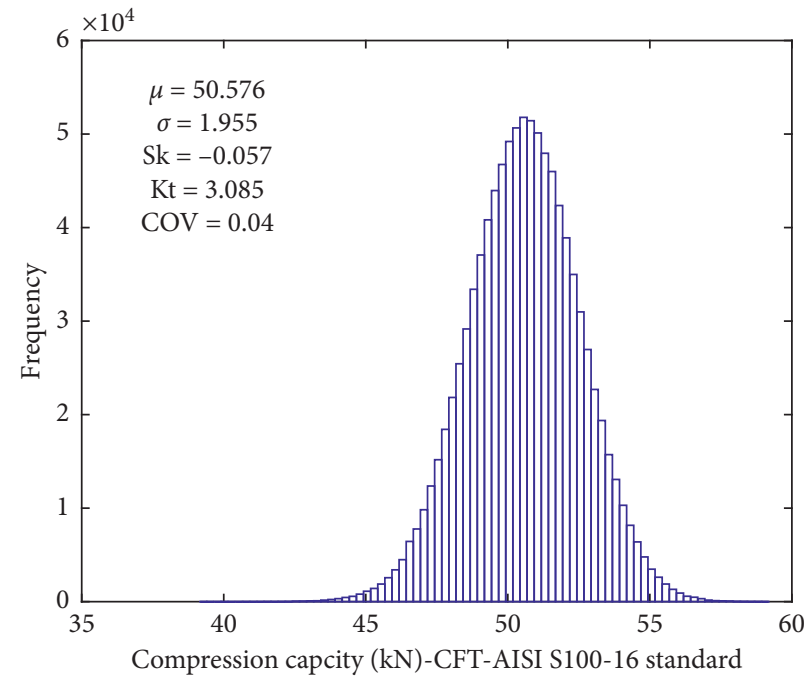

(a)

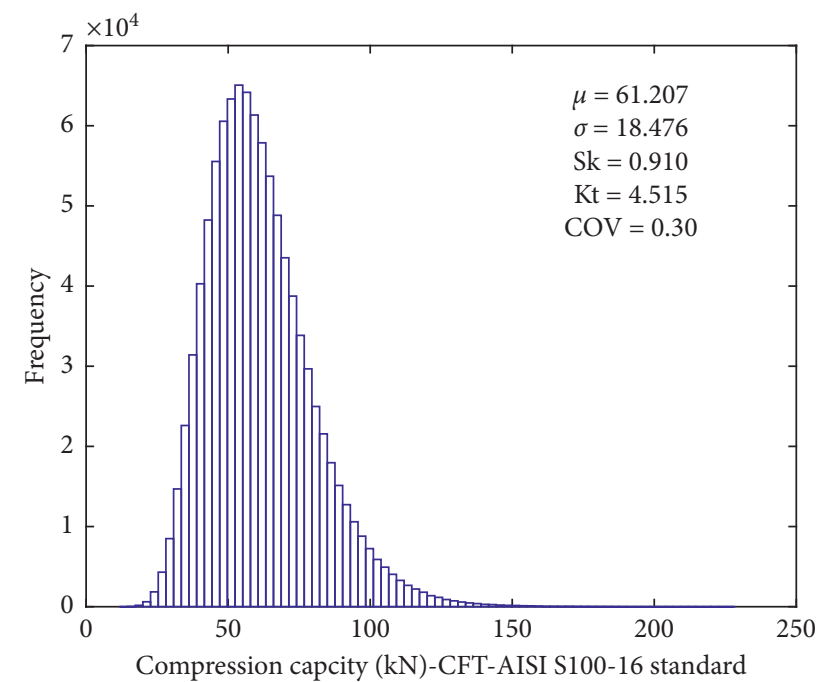

(b)

FIGURE 10: Compression capacity histograms for AISI S100-16 \& AS/NZS 4600: 2018 Standard: (a) compression capacity without ME; (b) compression capacity with ME. CFT—Column flexure-torsion buckling mode, $\mu-$ Mean, $\sigma-S t d$. deviation, Sk-Skewness, Kt—Kurtosis, and $\mathrm{COV}-\mathrm{Coefficient}$ of variation.

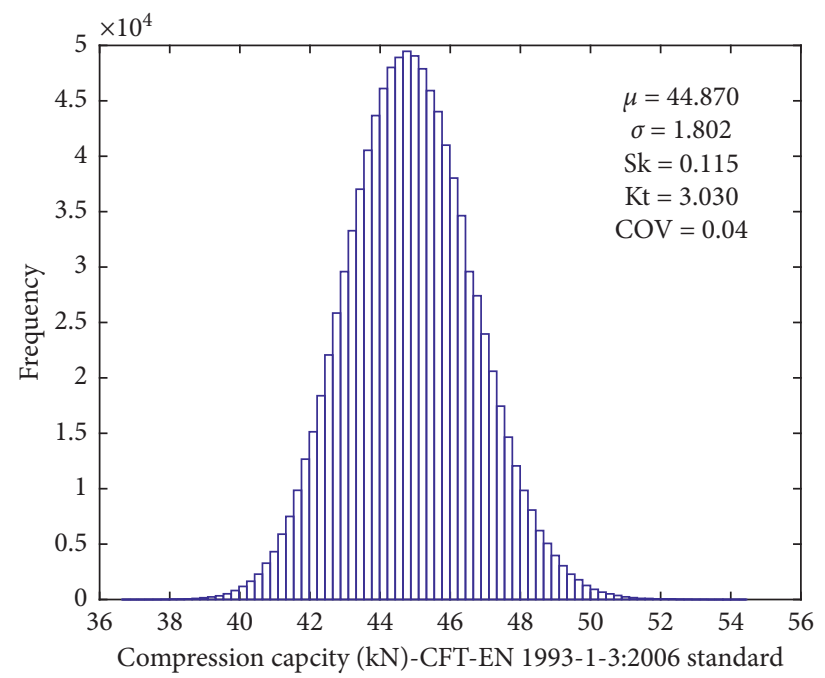

(a)

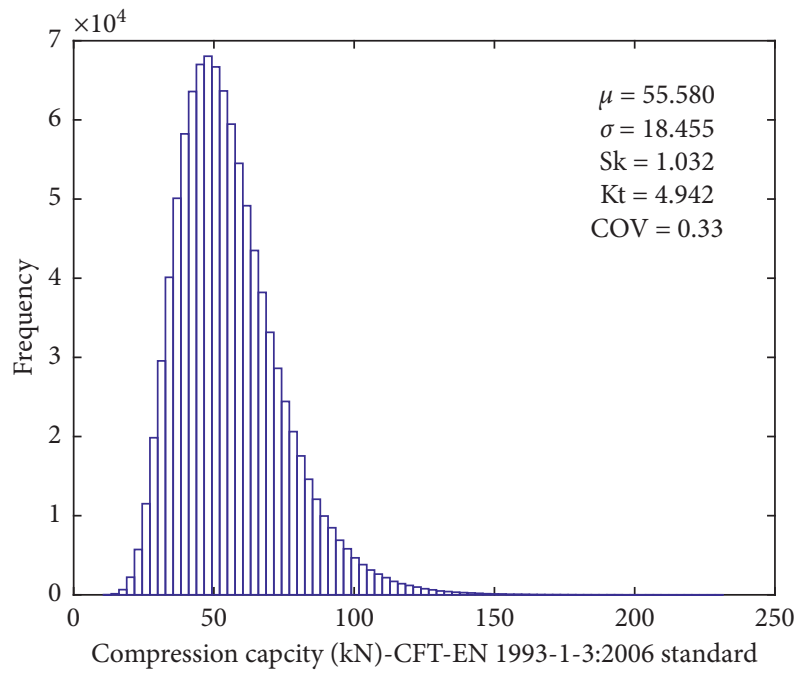

(b)

FIgURE 11: Compression capacity histograms for EN 1993-1-3:2006 Standard: (a) compression capacity without ME; (b) compression capacity with ME. CFT-Column flexure-torsion buckling mode, $\mu$-Mean, $\sigma-$ Std. deviation, Sk-Skewness, Kt-Kurtosis, and COV-Coefficient of variation.

1-3:2006 standard), negative skewness values are observed indicating negatively skewed or left skewed ME data. The close observation of test data of these failure modes indicates that the samples data points are less and require additional experimental investigations to get the correct statistics.

7.1.4. Kurtosis. The failure modes in flexure and flexuretorsion buckling for columns irrespective of the design standard, along with local buckling mode for column and stub columns as per AISI S100-16 \& AS/NZS 4600:2018 standard and combined local and distortion buckling mode as per EN 1993-1-3:2006 standard, kurtosis values are found to be more than three. This indicates non-Gaussian distribution for the ME data and the positive excess kurtosis in these cases indicate the data is leptokurtic having heavy tails and contains extreme values.

For other modes of failure, the kurtosis values are close to three with negative excess kurtosis which indicates that the data is platykurtic and having flat tails with small probability of extreme values. In this case, also the statistical distribution for ME data is nonGaussian.

7.1.5. Distribution. Chi-square goodness-of-fit tests are performed to determine the nature of probability 
TABLE 8: Results of Chi-square goodness-of-fit test for resistance simulation (database column no. 18-flexure-torsion mode of buckling).

\begin{tabular}{|c|c|c|c|c|c|c|}
\hline \multirow{2}{*}{ Design standard } & \multirow{2}{*}{ RVs } & \multirow{2}{*}{$\begin{array}{l}\text { Assumed distribution } \\
\text { (DOF) }\end{array}$} & \multirow{2}{*}{$\begin{array}{l}\text { Computed Chi-square } \\
\text { value }\end{array}$} & \multicolumn{2}{|c|}{$\begin{array}{l}\text { Allowable Chi- } \\
\text { square value }\end{array}$} & \multirow{2}{*}{$\begin{array}{l}\text { Null } \\
\text { hypothesis }\end{array}$} \\
\hline & & & & $\begin{array}{c}\text { At } \\
\alpha=5 \%\end{array}$ & $\begin{array}{c}\text { At } \\
\alpha=1 \%\end{array}$ & \\
\hline \multirow{3}{*}{ ASCE 10-15 (2015) } & $F_{\mathrm{y}}, E, \& \mathrm{ME}$ & Normal (77) & $\begin{array}{l}2.460 \times 10^{10} \\
7164 \times 10^{-5}\end{array}$ & 98.202 & 108.375 & Rejected \\
\hline & & Normal (77) & 0.0024 & & & Accepted \\
\hline & $F_{\mathrm{y}} \& \mathrm{E}$ & Lognormal (77) & $8.663 \times 10^{-5}$ & 98.202 & 108.375 & Accepted \\
\hline \multirow{3}{*}{$\begin{array}{l}\text { AISI S100-16 (2006) \& AS/NZS } \\
\text { 4600: } 2018(2018)\end{array}$} & $F_{\mathrm{y}}, F_{\mathrm{u}}, E, \&$ & Normal (77) & $2.032 \times 10^{7}$ & 98.202 & 108.375 & Rejected \\
\hline & $\mathrm{ME}$ & Lognormal (77) & $2.781 \times 10^{-4}$ & & & Accepted \\
\hline & $F_{\mathrm{y}}, F_{\mathrm{u}}, \& \mathrm{E}$ & Lognormal (77) & 0.0394 & 98.202 & 108.375 & $\begin{array}{l}\text { Accepted } \\
\text { Accepted }\end{array}$ \\
\hline \multirow{3}{*}{ EN 1993-1-3:2006 (2006) } & $F_{\mathrm{v}}, F_{\mathrm{u}}, E, \&$ & Normal (77) & $2.220 \times 10^{9}$ & & & Rejected \\
\hline & $\mathrm{ME}$ & Lognormal (77) & $8.753 \times 10^{-5}$ & 98.202 & 108.375 & Accepted \\
\hline & $F_{\mathrm{y}}, F_{\mathrm{u}}, \& \mathrm{E}$ & Normal (77) & 0.003 & 98.202 & 108.375 & Accepted \\
\hline
\end{tabular}

distribution of ME. Three different hypothetical distributions, namely, Normal, Lognormal, and Uniform distributions are considered for the test. The results of these tests are presented in Table 7. From the results of Chi-square goodness-of-fit test, it is found that the hypothesis of assumed distributions considered cannot be rejected at $1 \%$ and $5 \%$ of significance level. However, a distribution with the lowest Chi-square value is considered for the particular mode of failure. In some cases, the Normal distribution is governing but considering nonzero values of skewness and kurtosis value not equal to 3.0, lognormal distribution is assumed. The assumption of lognormal distribution for $\mathrm{ME}$ is also justified since negative values for $\mathrm{ME}$, however small they may be, do not have engineering meaning. The ME for the failure modes, for which the sample size is small, can be assumed to follow a uniform distribution.

7.2. Probabilistic Analysis of Compression Capacity. From Figures 9-11, it is observed that the distribution of compression capacities, without ME as random variable, follows normal distribution irrespective of design standard and the mean resistance value is equal to compression capacity calculated as per the respective design standards. However, the distribution of compression capacity, with $\mathrm{ME}$ as a random variable, seems to follow the lognormal distribution irrespective of design standard, and the COV for simulated compression capacity with $\mathrm{ME}$ as random variable is 0.3 , which is significant compared to COV of resistance without considering the ME.

The Chi-square goodness-of-fit test results presented in Table 8 justify the use of the distributions for resistance suggested earlier based on eye judgement.

A summary of conclusions are drawn and presented in the following section, based on the above discussions.

\section{Summary and Conclusions}

The model functions available for different modes of failure in ASCE 10-15, AISI S100-16, AS/NZS 4600:2018, and EN 1993-1-3:2006 standards are used to predict the compression capacity of a set of 273 CFS lipped channel compression members. The test data of compression members are obtained from the literature. Using these test results and also those of three nominally similar compression members tested at CSIR-SERC, a database is created for the first time and presented in this paper. Each test compression member has sufficient information for calculating the compression capacity using the abovementioned standards. Using the ratio of the test to predict compression capacity, $\mathrm{ME}$ analysis was carried out to assess the accuracy of model functions to calculate compression capacity for various failure modes available in these standards and to suggest probability distributions for the ME. The results of statistical analysis are briefly summarised as follows:

(i) From the results presented in Tables 6 and 7, it is inferred that the ME for various failure modes (except for the cases for which the data points are less), follows a lognormal distribution at 5\% significance level with means approximately equal to 1.10 and $\mathrm{COV}=0.25$. A higher value of $\mathrm{COV}$ is recommended to offset the effect of shield over estimation of capacity by ASCE 10-15 standard. In general, EN 1993-1-3:2006 standard seems to perform satisfactorily in the estimation of capacity of compression members. The values of coefficient of skewness and kurtosis, obtained in this investigation, also suggest the use of unsymmetrical distribution about mean (which in this paper is lognormal distribution).

(ii) It is also noted from Tables 6 and 7 that where the data points are less, the maximum entity distribution, i.e., uniform distribution, is recommended for ME.

(iii) As can be expected, the COV of ME associated with prediction models for combined failure modes are higher. This indicates that more number of tests to be carried out in this range for reducing statistical uncertainties.

(iv) The probabilistic analysis of compression capacity of CFS columns has brought out the importance of 
consideration of ME and in general, and it can be assumed that the compression capacity of column follows a lognormal distribution at 5\% significance level.

(v) The results presented in this paper are first of its kind, could help in carrying out the calibration studies for CFS columns, and may have potential reference to design practice.

\section{Data Availability}

The data used to support the findings of this study are included within the article as Table 4 and Figure 5.

\section{Disclosure}

This work was carried out as a part of $\mathrm{PhD}$ work of the first author at CSIR-SERC.

\section{Conflicts of Interest}

The authors declare that there are no conflicts of interest regarding the publication of this paper.

\section{Acknowledgments}

This paper is published with the kind permission of the Director, CSIR-SERC, Chennai, India.

\section{References}

[1] G. W. Juette and L. E. Zaffanella, "Radio noise, audible noise, and corona loss of EHY and UHV transmission lines under rain: predetermination based on cage tests," IEEE Transactions on Power Apparatus and Systems, vol. PAS-89, no. 6, pp. 1168-1178, 1970.

[2] A. Chajes, S. J. Britvec, and G. Winter, "Effects of coldstraining on structural steels," Journal of the Structural Division, ASCE, vol. 89, no. ST2, pp. 1-32, 1963.

[3] K. W. Karren, "Comer properties of cold-formed steel shapes," Journal of the Structural Division, ASCE, vol. 93, no. STl, 1967.

[4] K. W. Karren and G. Winter, "Effects of cold-work on light gage steel members," Journal of the Structural Division, ASCE, vol. 93, no. ST1, pp. 433-470, 1967.

[5] G. Winter and J. Uribe, "Effects of cold-work on cold-formed steel members," in Thin-Walled Steel Structures-Their Design and Use in Buildings, K. C. Rockey and H. V. Hill, Eds., Gordon and Breach Science Publishers, London, UK, 1968.

[6] S. R. Sathish Kumar and A. R. Santha Kumar, "Cold form steel," Lecture Notes on Design of Steel Structures II, IIT Madras, Chennai, India, 2009.

[7] E. H. Gaylord and G. M. Wilhoite, "Transmission towers: design of cold-formed angles," Journal of the Structural Division, vol. 111, no. 8, pp. 1810-1825, 1985.

[8] ASCE 10-15 Standard, Design of Latticed Steel Transmission Structures, American Society of Civil Engineers, Reston, VA, USA, 2015.

[9] AISI S100-16 Standard, North American Specification for the Design of Cold-Formed Steel Structural Members, American Iron and Steel Institute, , Washington, DC, USA, 2016.
[10] AS/NZS 4600:2018 Standard, Australian/New Zealand Standard Cod-Formed Steel Structures, SAI Global Limited under license from Standard Australia Limited, Sydney, Australia, 2001.

[11] EN 1993-1-3:2006 Standard, Eurocode 3: Design of Steel Structures-Part 1-3: General Rules-Supplementary Rules for Cold-Formed Members and Sheeting, vol. 36, European Committee for Standardization, Brussels, Belgium, 2006.

[12] IS: 801 (Draft Code), Code of Practice for the Use of Light Gauge Steel Structural Members in General Building Construction, Bureau of Indian Standards, New Delhi, India, 2010.

[13] JCSS Probabilistic Model Code, Part 3: Resistance Models-Model Uncertainties, JCSS, Technical University of Denmark, Aalborg, Denmark, 2001.

[14] C. C. Weng and T. Pekoz, "Compression tests of cold-formed steel columns," Journal of the Structural Division, ASCE, vol. 116, no. 5, pp. 1230-1246, 1990.

[15] C. C. Weng and C. P. Lin, "Study on maximum strength of cold-formed steel columns," Journal of the Structural Engineering, vol. 118, no. 1, pp. 128-146, 1992.

[16] D. T. Dat, "The strength of cold-formed steel columns," Report no. 80-4, Department of Structural Engineering, Cornell University, Ithaca, NY, USA, 1980.

[17] G. P. Mulligan, "The influence of local buckling on the structural behaviour of symmetric cold-formed steel columns," Report no. 83-1, Department of Structural Engineering, Cornell University, Ithaca, NY, USA, 1983.

[18] J. Loughlan, Mode interaction in lipped channel under concentric or eccentric loading, Ph.D thesis, Department of Mechanics of Materials, University of Strathclyde, Glasgow, Scotland, 1979.

[19] J. Loughlan and J. Rhodes, “The interactive buckling of lipped channel columns under concentric or eccentric loading," in Proceedings of the 1980 International Conference on Thin Walled Structures, Glasgow, Scotland, 1980.

[20] B. Young and K. J. R. Rasmussen, "Design of lipped channel columns," Journal of the Structural Engineering, vol. 124, no. 2, pp. $140-148,1998$.

[21] A. H. Chilver, "The stability and strength of thin-walled steel struts," The Engineer, vol. 196, pp. 180-183, 1953.

[22] T. Pekoz, "Post buckling interaction of plate elements," Progress report to Swedish Building Research Council, Department of Structural Engineering, Cornell University, Ithaca, NY, USA, 1977.

[23] J. Zaras and J. Rhodes, "Carefully controlled compression tests on thin-walled cold-formed sections," in Applied Solid Mechanics, A. S. Tooth and J. Spence, Eds., pp. 519-551, Cambridge University Press, Cambridge, UK, 1987.

[24] R. P. Rokade, N. Prasad Rao, R. Bala Gopal et al., "Studies on cold-formed lipped channel section for transmission line towers," in Proceedings of the 2010 National Conference on Latest Trends in Design and Testing of Transmission Line Components, pp. II-12-II-18, Bangalore, India, November 2010.

[25] T. H. Miller and T. Pekoz, "Unstiffened strip approach for perforated wall studs," Journal of the Structural Engineering, vol. 120, no. 2, pp. 410-421, 1994.

[26] A. Moldovan, "Compression tests on cold-formed steel columns with monosymmetrical section," Thin-Walled Structures, vol. 20, no. 1-4, pp. 241-252, 1994.

[27] K. S. Sivakumaran, "Load capacity of uniformly compressed cold-formed steel section with punched web," Canadian Journal of Civil Engineering, vol. 14, no. 4, pp. 550-558, 1987.

[28] P. Thomasson, "Thin-walled C-shaped panels in axial compression," Report no. D1:1978, Swedish Council for Building Research, Stockholm, Sweden, 1978. 
[29] S. M. Chou, L. K. Seah, and J. Rhodes, "The accuracy of some codes of practices in predicting the load capacity of coldformed columns," Journal of Constructional Steel Research, vol. 37, no. 2, pp. 137-172, 1996.

[30] Y. Pu, M. H. R. Godley, and R. G. Beale, "Experimental procedures for stub column tests," in Proceedings of the 14th International Specialty Conference on Cold-Formed Steel Structures, pp. 311-322, St. Louis, MO, USA., October 1998.

[31] M. Dundu, "Buckling of short cold-formed lipped channels in compression," Journal of The South African Institution of Civil Engineering, vol. 56, no. 2, pp. 46-53, 2014.

[32] T. C. H. Ting, K. Roy, H. H. Lau, and J. B. Lim, "Effect of screw spacing on behavior of axially loaded back-to-back coldformed steel built-up channel sections," Advances in Structural Engineering, vol. 21, no. 3, pp. 474-487, 2018.

[33] J. Whittle and C. Ramseyer, "Buckling capacities of axially loaded, cold-formed, built-up channels," Thin-Walled Structures, vol. 47, no. 2, pp. 190-201, 2009.

[34] J. Ye, I. Hajirasouliha, and J. Becque, "Experimental investigation of local-flexural interactive buckling of cold-formed steel channel columns," Thin-Walled Structures, vol. 125, pp. 245-258, 2018.

[35] J. Ye, S. M. Mojtabaei, and I. Hajirasouliha, "Local-flexural interactive buckling of standard and optimised cold-formed steel columns," Journal of Constructional Steel Research, vol. 144, pp. 106-118, 2018.

[36] K. Balaji Rao and T. V. S. R. Appa Rao, "Probabilistic analysis of strength of steel imperfect columns," Journal of Structural Engineering, vol. 27, no. 1, pp. 41-48, 2000.

[37] E. M. Lui and W.-F. Chen, "Simplified approach to the analysis and design of columns with imperfections," Engineering Journal, vol. 21, no. 2, pp. 99-117, 1984.

[38] J. Rondal and S. Maquio, "Single equation for SSRC column strength curves," Journal of Structural Engineering, vol. 105, no. ST1, pp. 247-250, 1979.

[39] L. E. Hsiao, W. W. Yu, and T. V. Galambos, "Load and resistance factor design of cold-formed steel: calibration of the AISI design provisions," 9th progress report, Civil Engineering Study 88-2, University of Missouri-Rolla, Rolla, MO, USA, 1988.

[40] T. N. Rang, T. V. Galambos, and W. W. Yu, "Load and resistance factor design of cold-formed steel: study of design formats and safety index combined with calibration of the AISI formulas for cold work and effective design width," 1 st progress report, Civil Engineering Study 79-1, University of Missouri- Rolla, Rolla, MO, USA, 1979.

[41] T. N. Rang, T. V. Galambos, and W. W. Yu, "Load and resistance factor design of cold-formed steel: statistical analysis of mechanical properties and thickness of material combined with calibration of the AISI design provisions on unstiffened compression elements and connections," 2nd progress report, Civil Engineering Study 79-2, University of Missouri-Rolla, Rolla, MO, USA, 1979.

[42] T. N. Rang, T. V. Galambos, and W. W. Yu, "Load and resistance factor design of cold-formed steel: calibration of the design provisions on connections and axially loaded compression members," 3rd progress report, Civil Engineering Study 79-3, University of Missouri-Rolla, Rolla, MO, USA, 1979.

[43] T. N. Rang, T. V. Galambos, and W. W. Yu, "Load and resistance factor design of cold-fonned steel: calibration of the design provisions on laterally unbraced beams and beamcolumns," 4th progress report, Civil Engineering Study 79-4, University of Missouri-Rolla, Rolla, MO, USA, 1979.

[44] B. Supornsilaphachai, T. V. Galambos, and W. W. Yu, "Load and resistance factor design of cold-formed steel: calibration of the design provisions on beam webs," Fifth progress report, Civil Engineering Study 79-5, University of Missouri-Rolla, Rolla, MO, USA, 1979.

[45] B. Ellingwood, T. V. Galambos, I. G. MacGregor et al., Development of a Probability Based Load Criterion for American National Standard A58: Building Code Requirements for Minimum Design Loads in Buildings and Other Structures, vol. 577, U.S. Department of Commerce, National Bureau of Standards, NBS Special Publication, Gaithersburg, MD, USA, 1980.

[46] T. V. Galambos, B. Ellingwood, J. G. MacGregor et al., "Probability based load criteria: assessment of current design practice," Journal of the Structural Division, ASCE, vol. 108, no. ST5, 1982.

[47] B. Ellingwood, J. G. MacGregor, T. V. Galambos et al., "Probability based load criteria: load factors and load combinations," Journal of the Structural Division, ASCE, vol. 108, no. ST5, 1982.

[48] I.S.: 811, Specification for Cold Formed Light Gauge Structural Steel Sections, Bureau of Indian Standards, New Delhi, India, 2004.

[49] I.S.: 1852, Specifications for Rolling and Cutting Tolerances for Hot-Rolled Steel Products, Bureau of Indian Standards, New Delhi, India, 2003.

[50] E8/E8M-15a, Standard Test Methods for Tension Testing of Metallic materials, ASTM International, West Conshohocken, PA, USA, 2015.

[51] E6 -15, Standard Terminology Relating to Methods of Mechanical Tests, ASTM International, West Conshohocken, PA, USA, 2016.

[52] C. T. F. Ross, Mechanics of Solids, Horwood Publishing Limited, International Publishers, Coll House, Chichester, UK, 1999.

[53] G. Winter, Commentary on the 1968 Edition of the Specification for the Design of Cold-Formed Steel Structural Members, American Iron and Steel Institute, New York, NY, USA, 1970.

[54] A. Chajes and G. Winter, "Torsional-flexural buckling of thinwalled members," Journal of the Structural Division, ASCE, vol. 91, no. ST4, 1965.

[55] A. Chajes, P. J. Fang, and G. Winter, "Torsional-flexural buckling, elastic and inelastic, of cold-formed thin-walled columns," Engineering Research Bulletin, vol. 66, 1966.

[56] W.-W. Yu and R. A. LaBoube, Cold-Formed Steel Design, John Wiley \& Sons, Inc., Hoboken, NJ, USA, 4th edition, 2010.

[57] B. W. Schafer, "Local, distortional and Euler buckling of thin walled columns," Journal of Structural Engineering, vol. 128, no. 3, pp. 289-299, 2002.

[58] C. Yu and B. W. Schafer, "Distortional buckling tests on coldformed steel beams," Journal of Structural Engineering, vol. 132, no. 4, pp. 515-528, 2006.

[59] M. K. Ravindra and T. V. Galambos, "Load and resistance factor design for steel," Journal of the Structural Division, ASCE, vol. 104, no. ST9, pp. 1459-1468, 1978.

[60] P. E. Hess, D. Bruchman, A. Ibrahim et al., "Uncertainties in material and geometric strength and load variables," Naval Engineers Journal, vol. 114, no. 2, pp. 139-166, 2002. 